TRANSACTIONS OF THE

AMERICAN MATHEMATICAL SOCIETY

Volume 353, Number 12, Pages 5121-5144

S 0002-9947(01)02797-0

Article electronically published on July 13, 2001

\title{
GEOMETRIC REPRESENTATION OF SUBSTITUTIONS OF PISOT TYPE
}

\author{
VINCENT CANTERINI AND ANNE SIEGEL
}

\begin{abstract}
We prove that a substitutive dynamical system of Pisot type contains a factor which is isomorphic to a minimal rotation on a torus. If the substitution is unimodular and satisfies a certain combinatorial condition, we prove that the dynamical system is measurably conjugate to an exchange of domains in a self-similar compact subset of the Euclidean space.
\end{abstract}

\section{INTRODUCTION}

In [27, G. Rauzy proves that the dynamical system generated by the substitution $\sigma(1)=12, \sigma(2)=13, \sigma(3)=1$, is measure-theoretically conjugate to an exchange of domains in a compact subset $\mathcal{R}$ of the complex plane. This compact subset has a self-similar structure: using methods introduced by F. M. Dekking in [11], S. Ito and M. Kimura obtain in [19] an alternative construction of $\mathcal{R}$ and prove that each of the exchanged domains has fractal boundary. In the same paper they also study the Hausdorff dimension of the boundary of $\mathcal{R}$. A. Messaoudi in 24] obtains additional properties of the fractal $\mathcal{R}$ including boundary parametrization.

By studying the numeration system of the Tribonacci number (root of the polynomial $x^{3}-x^{2}-x-1$ of modulus smaller than one), G. Rauzy proves that $\mathcal{R}$ projects onto the torus in a measure-theoretically one-to-one way. This implies that the compact set $\mathcal{R}$ generates a periodic tiling of the plane with fractal boundary. The self-similarity of $\mathcal{R}$ implies that the induced tiling is quasi-periodic. This is analogous to the well understood connection between sturmian sequences, tilings of the line and the structure of quasi-crystals (see [1]), and more generally to the theory of quasi-crystals: via methods similar to those used by G. Rauzy in [27, E. Bombieri and J. Taylor use substitutions to exhibit a connection between quasicrystals and number theory in [5].

From the point of view of ergodic theory, the result of G. Rauzy means that the subshift generated by the substitution $\sigma$ is measure-theoretically conjugate to a rotation on the 2-torus. M. Queffélec defines in [26] (page 140) a geometric representation of a symbolic system $(\Omega, S)$ as a geometrical dynamical system $(\mathcal{X}, T)$, on which there exists a partition indexed by the alphabet, such that every word in $\Omega$ is the itinerary of a point of $(\mathcal{X}, T)$ with respect to the partition. She asks which systems admit such a geometric representation.

Received by the editors February 1, 2000 and, in revised form, August 12, 2000.

2000 Mathematics Subject Classification. Primary 37B10, 28A80; Secondary 47A35.

Key words and phrases. Substitutive dynamical system, toral translation, factor map, domain exchange. 
There have been many partial answers given to Queffélec's question: in [3], G. Rauzy and P. Arnoux study a class of uniformly recurrent sequences of block complexity $2 n+1$ satisfying a certain combinatorial criterion (the so-called $*$ condition of Arnoux-Rauzy) and show that each such sequence codes the orbit of a point under an exchange of six intervals on the circle. In [6], M. Boshernitzan and I. Kornfeld study the dynamics of a self-similar interval translation mapping which is measure isomorphic to a subshift generated by a substitution. In [12], S. Ferenczi obtains a geometric realization of the Chacon sequence as exduction of a triadic rotation. In [15, 16] C. Holton and L. Zamboni define real and complex representations associated to each substitution whose occurrency matrix has a nonzero eigenvalue of modulus less than one.

From the point of view of spectral theory, representing a substitutive system by a translation means than the coordinates of the translation vector are eigenvalues for the unitary operator associated to the shift map, each coordinate of the representation map being an eigenfunction of the unitary operator. Thus, looking for continuous representations of a substitutive system as toral translations is strongly linked with the determination of the dynamical system's spectrum and its continuous eigenfunctions.

B. Host has made a significant advance in this subject by proving that all eigenfunctions of primitive substitutive dynamical systems are continuous [17]. Thus, very happily, the two main dynamical classifications (up to measure-theoretic and topological isomorphisms) are equivalent for primitive substitutive systems. Moreover, B. Host [17, 18, A. N. Livshits [23], B. Solomyak [32, have given conditions for a substitutive dynamical system to have discrete spectrum. Some are necessary conditions, others are sufficient. In particular, B. Host and independently A. N. Livshits (whose work was restated and generalized by M. Hollander) defined a combinatorial condition on two-letter substitutions called the coincidence condition. This condition generalizes a definition of F. M. Dekking [10] for constant length substitutions. M. Hollander [13] proved that this condition is equivalent to the fact that a two-letter primitive substitutive system has a discrete spectrum (see the survey in [30]). This combinatorial condition was generalized to all substitutions by P. Arnoux and S. Ito in [2]. It is conjectured that every substitution of Pisot type satisfies the coincidence condition, but this is still unknown, even for $d=2$.

Nevertheless, an explicit description of the spectrum and the eigenfunctions of substitutive systems was obtained only for a few examples. In 32, B. Solomyak shows that the substitution on $d$ letters defined by $\sigma(1)=12 \ldots d, \sigma(2)=1$, $\sigma(3)=2, \ldots, \sigma(d)=d-1$, may be realized as an explicit translation on the $(d-1)$-dimensional torus. In 33, M. Solomyak proves that a class of unimodular substitutions of Pisot type on $d$ letters admit as a metric factor a translation on the $(d-1)$-dimensional torus. B. Host, in an unfortunately unpublished work [18, explicitly realizes every substitution of Pisot type on 2 letters as a translation on the 1-torus. In contrast, J. Cassaigne, S. Ferenczi and L. Zamboni exhibit an example of an Arnoux-Rauzy sequence on 3-letters which cannot be geometrically realized as a rotation on the $n$-torus 9 .

In this paper we generalize the results of G. Rauzy, M. Solomyak and B. Host: for all substitutive dynamical systems of Pisot type, we give an explicit continuous semi-conjugacy between the shift on the system and a translation on a torus. Unlike most of the above mentioned papers, our semi-conjugacy is defined everywhere and 
not just on a set of full measure. This allows us to give a new proof of the results in [32, 33] which makes the representation explicit.

In Section 1 we recall some of the results in [8] and [16] on the connection between primitive substitutive systems and subshifts of finite type. Indeed, primitivity of the substitution implies that it is possible to map any point of the system to a path in an automaton, in a unique way except on a countable subset. This map measure-theoretically conjugates the shift map on the substitutive system with an adding machine on the subshift of finite type, considered as a Markov compactum in which the order is naturally defined by the substitution.

If the substitution is of Pisot type on a $d$ letters alphabet, this coding allows us in Section 2 to realize any element of $\Omega$ as a point in $\mathbb{R}^{d-1}$. On each coordinate, this mapping coincides with the real or complex representations of C. Holton and L. Zamboni in 15. Gathering all the one-dimensional realizations together is natural in the sense that each is a different expression of the same formal numeration system (the one generated by the characteristic polynomial of the occurrency matrix of the substitution).

We prove in Section 3 that this representation map projects onto the $(d-1)$-torus and satisfies a commutation relationship with a translation.

Theorem. Every dynamical system generated by a substitution of Pisot type on $d$ letters admits as a topological factor a minimal translation on the torus $\mathbb{T}^{d-1}$.

In [2], P. Arnoux and S. Ito associate to any unimodular substitution of Pisot type on $d$ letters a set in $\mathbb{R}^{d-1}$ called Rauzy fractal, which can be naturally divided in $d$ domains. They prove that when the domains are disjoint up to a set of measure zero, one can define an exchange of domains on the Rauzy fractal which is measure-theoretically conjugate to the shift map on $\Omega$. They also prove that a sufficient condition for the domains to be disjoint in measure is that the substitution satisfies the combinatorial coincidence condition.

In Section 4, we use the representation defined in Section 2 to give a new proof of P. Arnoux and S. Ito theorem.

Theorem. If $\sigma$ is a substitution on d letters, of Pisot type, unimodular, and satisfying the coincidence condition, the symbolic dynamical system generated by $\sigma$ is measure-theoretically conjugate to an exchange of domains in a self-similar compact subset of $\mathbb{R}^{d-1}$.

The self-similar compact subset of $\mathbb{R}^{d-1}$ we get is exactly the Rauzy fractal associated to the substitution in [2]. The interest of our methods is that the Rauzy fractal is obtained as the image of an explicit map, so that it makes it possible to derive various topological properties of the fractal. For instance, the surjectivity of the toral representation yields a new proof of the fact that the fractal has a nonempty interior, which was first proved by V. Sirvent and Y. Wang in [31]. Moreover, the first author gives in 7] a criterion for the connectedness of the fractal.

In [30], the second author defines a criterion for the injectivity in measure of the toral realization for unimodular substitutions. When this criterion is satisfied, the results and methods contained in this paper can be used to obtain explicit fractal tilings of the plane and produce Markov partitions for Pisot toral automorphisms, generalizing the work of A. Messaoudi in [24] about the Rauzy substitution. The existence of such Markov partitions is established by R. Kenyon and A. Vershik in [22], although their proof is not constructive. The formalism of substitutions 
induces a natural order on the Markov compactum and yields a Markov partition which is explicit, so that its topological properties can be studied (boundary, connectedness, simply connectedness).

In the nonunimodular case, it is sometimes possible to complete the representation by translations on $p$-adic groups $\mathbb{Z}_{p}$ : this depends on the ramifications of the determinant of the incident matrix of $\sigma$ in the Galois extension of its dominant eigenvalue [29].

Acknowledgments. The authors wish to thank P. Arnoux for his guidance and for many useful comments and suggestions. We also wish to thank B. Host for sharing with us his profound insight in the subject. Finally we wish to thank V. Berthé and L. Q. Zamboni for their attention and support in the preparation of the manuscript.

\section{Generalities}

1.1. Substitutive dynamical systems. Let $\mathcal{A}$ be a finite alphabet, $\mathcal{A}^{*}$ the set of finite words in $\mathcal{A}$ and $\mathcal{A}^{+}$the subset of $\mathcal{A}^{*}$ of nonempty words. The empty word is denoted $\varepsilon$.

A two-sided sequence in $\mathcal{A}$, also called doubly infinite word, is denoted $w=$ $\ldots w_{-2} w_{-1} \cdot w_{0} w_{1} \ldots$ The topology of the set of doubly infinite words $\mathcal{A}^{\mathbb{Z}}$ will be the product of the discrete topology on $\mathcal{A}$. This topology is metrizable. A cylinder of $\mathcal{A}^{\mathbb{Z}}$ is a clopen set of the form:

$$
\left[U_{1} . U_{2}\right]=\left\{\left(w_{i}\right)_{i} \in \mathcal{A}^{\mathbb{Z}} \mid w_{-\left|U_{1}\right|} \ldots w_{-1} w_{0} \ldots w_{\left|U_{2}\right|-1}=U_{1} U_{2}\right\}, \quad U_{1}, U_{2} \in \mathcal{A}^{*}
$$

(if $U_{1}$ is empty, the cylinder is denoted $\left[U_{2}\right]$ ); the cylinders form a basis of the topology on $\mathcal{A}^{\mathbb{Z}}$.

A substitution $\sigma$ is an endomorphism of the free-monoid $\mathcal{A}^{*}$, such that the image of each letter of $\mathcal{A}$ is nonempty, and that for at least one letter $a$, we have $\lim _{n \rightarrow \infty}\left|\sigma^{n}(a)\right|=+\infty$. A substitution naturally extends to the set of doubly infinite words $\mathcal{A}^{\mathbb{Z}}$ :

$$
\sigma\left(\ldots w_{-2} w_{-1} . w_{0} w_{1} \ldots\right)=\ldots \sigma\left(w_{-2}\right) \sigma\left(w_{-1}\right) . \sigma\left(w_{0}\right) \sigma\left(w_{1}\right) \ldots
$$

A doubly infinite word $u=\left(u_{i}\right)_{i \in \mathbb{Z}}$ in $\mathcal{A}^{\mathbb{Z}}$ such that there exists a positive integer $k$ with $\sigma^{k}(u)=u$ is called a periodic point of $\sigma$. If $\sigma(u)=u$, then $u$ is a fixed point of $\sigma$. Every substitution has at least one periodic point (see [26]).

Denote by $S$ the shift map on $\mathcal{A}^{\mathbb{Z}}$ :

$$
S\left(\left(w_{i}\right)_{i \in \mathbb{Z}}\right)=\left(w_{i+1}\right)_{i \in \mathbb{Z}} .
$$

A word $w$ in $\mathcal{A}^{\mathbb{Z}}$ such that there exists a positive integer $h$ with $S^{h}(w)=w$ is called $S$-periodic. A substitution is said to be $S$-periodic when there exists a periodic point of $\sigma$ which is also $S$-periodic.

The language $\mathcal{L}(w)$ of a doubly infinite word $w$ is the set of finite words which appear in $w$. The symbolic dynamical system generated by a word $u$ is the pair $(\Omega(u), S)$, with $\Omega(u)=\left\{w \in \mathcal{A}^{\mathbb{Z}}, \mathcal{L}(w) \subset \mathcal{L}(u)\right\}$. Note that $\Omega(u)$ is the closure in $\mathcal{A}^{\mathbb{Z}}$ of the shift orbit of $u$, and that $\Omega(u)$ is finite if and only if $u$ is $S$-periodic. This set is a compact subset of $\mathcal{A}^{\mathbb{Z}}$, and $S$ is an homeomorphism on $\Omega(u)$.

We call a substitution primitive if there exists $k$ such that $\sigma^{k}(a)$ contains at least one occurrence of $b$ for every pair of letters $(a, b), k$ being independent of the letters. If $\sigma$ is primitive and $u$ is a periodic point of $\sigma$, then $\Omega(u)$ does not depend on $u$ and we denote by $(\Omega, S)$ the symbolic dynamical system generated by $\sigma$. 
If the substitution is primitive, the system $(\Omega, S)$ is a minimal dynamical system: it has no non-trivial closed $S$-invariant subset (see [26]). This system is uniquely ergodic: there exists a unique $S$-invariant probability measure on $\Omega$, denoted by $\mu_{\Omega}$. The measure of a cylinder $\left[U_{1} . U_{2}\right]$ is the frequency of the finite word $U_{1} U_{2}$ in any element of $\Omega$.

In the primitive case, one can remark the system $(\Omega, S)$ is finite if and only if the substitution is $S$-periodic. From here on, all substitutions are assumed to be primitive and non- $S$-periodic.

1.2. Substitutions of Pisot type. If the alphabet $\mathcal{A}$ is $\mathcal{A}=\{1, \ldots, d\}$, let $\mathbf{1}$ : $\mathcal{A}^{*} \mapsto \mathbb{N}^{d}$ be the natural homomorphism obtained by abelianization of the free monoid: if $|W|_{a}$ denote the number of occurrences of the letter $a \in \mathcal{A}$ in a finite word $W$, then we have $\mathbf{l}(W)=\left(|W|_{i}\right)_{i=1, \ldots, d} \in \mathbb{N}^{d}$.

Note that $d$-tuples are considered as column vectors in this paper.

To each substitution $\sigma$ on $\mathcal{A}$ is canonically associated its abelianized linear map whose matrix $\mathbf{M}_{\sigma}=\left(m_{i, j}\right)_{1 \leq i, j \leq d}$ (called occurrency matrix of $\sigma$ ) is defined by $m_{i, j}=|\sigma(j)|_{i}$, so that we have

$$
\forall W \in \mathcal{A}^{*} \quad \mathbf{l}(\sigma(W))=\mathbf{M}_{\sigma} \mathbf{l}(W) .
$$

A matrix $\mathbf{M}$ is said to be primitive if there exists a power of $\mathbf{M}$ whose coefficients are strictly positive. The formula (1.1) implies that a substitution is primitive if and only if its matrix is primitive. If $\sigma$ is primitive, the Perron-Frobenius theorem says that there exists a real positive eigenvalue $\alpha$ which is simple and strictly greater than the modulus of all the other eigenvalues. One can refer to [26] for more details about all those definitions.

Definition 1.1. An algebraic integer is a Pisot-Vijayaraghavan number or a Pisot number if all its algebraic conjugates $\beta$ verify $|\beta|<1$.

A substitution $\sigma$ is of Pisot type if its characteristic polynomial $P_{\sigma}$ is irreducible over $\mathbb{Q}$ and has a root $\alpha$ which is a Pisot number.

A substitution of Pisot type $\sigma$ is unimodular if $\operatorname{det} \mathbf{M}_{\sigma}= \pm 1$.

The next proposition gives a result about the eigenvalues of $\mathbf{M}_{\sigma}$.

Proposition 1.2. Let $\sigma$ be a primitive substitution and $P_{\sigma}$ the characteristic polynomial of the occurrency matrix $\mathbf{M}_{\sigma}$.

The substitution $\sigma$ is of Pisot type if and only if every nondominant root of $P_{\sigma}$ verifies $0<|\beta|<1$.

If $\sigma$ is of Pisot type, then $\sigma$ cannot be of constant length, the roots of $P_{\sigma}$ are all simple, and the matrix $\mathbf{M}_{\sigma}$ is diagonalizable with simple eigenvalues.

Proof. From the Gauss lemma, $P_{\sigma}$ is irreducible over $\mathbb{Q}$ if and only if it is irreducible over $\mathbb{Z}$. If $\sigma$ is not, there exists two polynomials $Q$ and $R$ with integer coefficients such that $P_{\sigma}=Q R$, then $Q$ and $R$ each have at least one root whose modulus is greater than 1, so that $P_{\sigma}$ has at least two roots of modulus greater than 1 . Two of those roots are different since the dominant eigenvalue is simple.

Consequently, if every nondominant root of $P_{\sigma}$ verifies $0<|\beta|<1$, then $P_{\sigma}$ is irreducible over $\mathbb{Q}$ and $\sigma$ is of Pisot type. Conversely, if $\sigma$ is of Pisot type, $P_{\sigma}$ is irreducible over $\mathbb{Q}$ so that 0 is not a root of $P_{\sigma}$ and every nondominant root of $P_{\sigma}$ verifies $0<|\beta|<1$. 
If $\sigma$ is of constant length $l$, that is the image of each letter contains exactly $l$ letters, then $l$ is an eigenvalue for the eigenvector $(1, \ldots, 1)$ and $P_{\sigma}$ is reducible over $\mathbb{Q}$.

This proposition implies that every substitution of Pisot type is primitive:

Proposition 1.3. Every substitution of Pisot type is primitive.

Proof. Let $\sigma$ be a substitution of Pisot type and $\mathbf{M}_{\sigma}$ be its occurrency matrix. We need to to prove that $\mathbf{M}_{\sigma}$ is a primitive matrix.

By definition of substitutions of Pisot type, $P_{\sigma}$ is irreducible over $\mathbb{Q}$. This implies that $\mathbf{M}_{\sigma}$ is an irreducible matrix: if $\mathbf{M}_{\sigma}$ is reducible, there exists a permutation matrix $\mathbf{P}$ such that

$$
\mathbf{P M}_{\sigma}{ }^{t} \mathbf{P}=\left(\begin{array}{cc}
B & 0 \\
D & C
\end{array}\right)
$$

is a block-triangular matrix with integer entries, and the characteristic polynomial $P_{\sigma}$ is reducible over $\mathbb{Z}$.

By a classic theorem (see for instance [4]), for a nonnegative matrix $A$, the following conditions are equivalent: (1) $A$ is primitive, (2) $A$ is irreducible and $\rho(A)$ is greater in magnitude than any other eigenvalue.

Since $\sigma$ is of Pisot type, $\rho\left(M_{\sigma}\right)$ is a Pisot number and then is greater in magnitude than any other eigenvalue.

In [15], C. Holton and L. Zamboni prove that a substitution of Pisot type cannot be $S$-periodic:

Proposition 1.4 (see [15]). If $\sigma$ is a primitive substitution whose matrix has an eigenvalue of modulus strictly smaller than 1, then any fixed point $u$ of $\sigma$ is not S-periodic.

Thus, the symbolic dynamical system associated with a substitution of Pisot type is well-defined and uniquely ergodic.

1.3. Coding of the symbolic dynamical system. Let $\sigma$ be a primitive and non$S$-periodic substitution. Our choice of considering the dynamical system consisting of sequences indexed by $\mathbb{Z}$ instead of $\mathbb{N}$ comes from the fact that every primitive substitution satisfies a property of bilateral recognizability while not every primitive substitution satisfies the property of unilateral recognizability. Indeed, in [25] B. Mossé proves that every word $w$ in $\Omega$ has a unique decomposition $w=S^{k}(\sigma(y))$, with $y \in \Omega$ and $0 \leq k<\left|\sigma\left(y_{0}\right)\right|$, where $y_{0}$ is the $0^{t h}$ coordinate of $y$. This means that any word of the dynamical system can be uniquely written on the form

$$
w=\ldots|\underbrace{\ldots}_{\sigma\left(y_{-1}\right)}| \underbrace{w_{-k} \ldots w_{-1} \cdot w_{0} \ldots w_{l}}_{\sigma\left(y_{0}\right)}|\underbrace{\ldots}_{\sigma\left(y_{1}\right)}| \underbrace{\ldots}_{\sigma\left(y_{2}\right)} \mid \ldots
$$

with $\ldots y_{-n} \ldots y_{-1} y_{0} y_{1} \ldots y_{n} \ldots$ in $\Omega$.

Denote $p=w_{-k} \ldots w_{-1}$ the prefix of $\sigma\left(y_{0}\right)$ of length $k$ and $s$ the suffix $s=$ $w_{1} \ldots w_{l}$. The word $w$ is completely defined by the word $y$ and the decomposition of $\sigma\left(y_{0}\right)$ on the form $p w_{0} s$. Let $\mathcal{P}$ be the finite set of all such decompositions:

$$
\mathcal{P}=\left\{(p, a, s) \in \mathcal{A}^{*} \times \mathcal{A} \times \mathcal{A}^{*} ; \exists b \in \mathcal{A} \text { and } \sigma(b)=p a s\right\} .
$$


The work of B. Mossé implies that we can define on one hand a desubstitution map $\theta$ on $\Omega$ (which sends $w$ to $y$ ), and a partition map $\gamma$ from $\Omega$ to $\mathcal{P}$, corresponding to the decomposition of $\sigma\left(y_{0}\right)$. These two maps are continuous.

$$
\begin{array}{cl}
\theta: \Omega \rightarrow \Omega, w \mapsto y & \text { such that } w=S^{k} \sigma(y) \text { and } 0 \leq k<\left|\sigma\left(y_{0}\right)\right|, \\
\gamma: \Omega \rightarrow \mathcal{P}, w \mapsto\left(p, w_{0}, s\right) & \text { such that } \sigma\left(y_{0}\right)=p w_{0} s \text { and } k=|p| .
\end{array}
$$

In [8] the itineraries of the points of $\Omega$ through the desubstitution according to the partition defined by $\gamma$ are studied.

Definition 1.5. We call the map $\Gamma: \Omega \rightarrow \mathcal{P}^{\mathbb{N}}$ which maps a word $w$ in $\Omega$ to the sequence $\left(\gamma\left(\theta^{i} w\right)\right)_{i \geq 0}$ in $\mathcal{P}^{\mathbb{N}}$ the prefix-suffix development

$$
\Gamma(w)=\left(\gamma\left(\theta^{i} w\right)\right)_{i \geq 0}=\left(p_{i}, a_{i}, s_{i}\right)_{i \geq 0} .
$$

Definition 1.6. The prefix-suffix automaton of the substitution $\sigma$ has $\mathcal{A}$ as a set of vertices and $\mathcal{P}$ as a set of label edges. There is an edge $e=(p, a, s)$ from $a$ to $b$ if and only if pas $=\sigma(b)$.

The set of labels of infinite walks in this automaton is denoted $\mathcal{D}$.

A consequence of $\sigma$ primitivity is that this automaton is strongly connected. We prove in [8] that $\Gamma$ is a measure-theoretic isomorphism between $\Omega$ and $\mathcal{D}$, similar results being obtained independently in [16]:

Theorem 1.7 (see [8, 16]). Let $\sigma$ be a primitive non-S-periodic substitution and $(\Omega, S)$ the dynamical system generated by $\sigma$. The map $\Gamma$ defined above is continuous and onto the subshift of finite type $\mathcal{D}$. This map is one-to-one except on the orbit of periodic points of $\sigma$.

This implies that the sets $S^{n} \sigma^{k}[i]$ partition $\Omega$ :

Corollary 1.8 (see [8]). For every $k \in \mathbb{N}$, let $\mathcal{P}_{k}=\left\{S^{n} \sigma^{k}[a] ; n \in \mathbb{N}, a \in \mathcal{A}, n<\right.$ $\left.\left|\sigma^{k}(a)\right|\right\}$. The sequence $\left(\mathcal{P}_{k}\right)_{k \in \mathbb{N}}$ of partitions of $\Omega$ is generating in measure.

More precisely, there exists a countable set $\mathcal{N}$ such that for every $k \in \mathbb{N}, \Omega \backslash \mathcal{N}=$ $\bigcup_{A \in \mathcal{P}_{k}} A \backslash \mathcal{N}$, the union being disjoint. Every sequence $\left(A_{k}\right)_{k \geq 0}$ with $A_{k} \in \mathcal{P}_{k}$ is such that $\cap A_{k}$ consists either in zero or one point, or in a finite number of points of $\mathcal{N}$.

We prove in 8 that the action of the desubstitution map $\theta$ on $\Omega$ is conjugate via $\Gamma$ with the map shift on $\mathcal{D}$. The action of the substitution map $\sigma$ on $\Omega$ is conjugate with the left extension of elements of $\mathcal{D}$ by an element of $\mathcal{P}$ which has an empty prefix. Finally, the shift map $S$ on $\Omega$ is conjugate with an adic transformation on $\mathcal{D}$. More precisely, we will need the following result:

Lemma 1.9 (see [8]). Let $w$ in $\Omega$ and $\Gamma(w)=\left(p_{i}, a_{i}, s_{i}\right)_{i \geq 0}$ its prefix-suffix development. The development $\Gamma(\sigma w)=\left(q_{i}, b_{i}, t_{i}\right)_{i \geq 0}$ is such that

$$
\forall i \geq 0 \quad q_{0}=\varepsilon \quad \text { and } \quad q_{i+1}=p_{i} .
$$

If $S w$ is a periodic point of $\sigma$ and $\Gamma(S w)=\left(r_{i}, c_{i}, u_{i}\right)_{i \geq 0}$ its development, then the sequence $\left(p_{i}\right)_{i \geq 0}$ is periodic, and the sequence $\left(r_{i}\right)_{i \geq 0}$ is the sequence of empty word $\varepsilon$.

If $S w$ is not periodic for $\sigma, \Gamma(S w)=\left(r_{i}, c_{i}, u_{i}\right)_{i \geq 0}$ is such that there exists an integer $n_{0}$ with

$$
\forall n \geq n_{0} \quad \sigma^{n}\left(p_{n}\right) \ldots \sigma^{0}\left(p_{0}\right) a_{0}=\sigma^{n}\left(r_{n}\right) \ldots \sigma^{0}\left(r_{0}\right) .
$$




\section{Representation of a substitutive system of Pisot type}

In this section, as in [27], the prefix-suffix development and an arithmetic normalization of the eigenvectors of the matrix of $\sigma$ are used to represent a substitutive system of Pisot type on a hyperplane.

2.1. Digit vectors for substitutions of Pisot type. Let $\sigma$ be a substitution of Pisot type, $\mathbf{M}_{\sigma}$ the matrix associated to $\sigma$, and $P_{\sigma}$ its characteristic polynomial.

We denote by $\alpha$ the dominant eigenvalue of $\mathbf{M}_{\sigma}$, by $\alpha_{1}=\alpha, \alpha_{2}, \ldots, \alpha_{r}$ the $r$ real roots of the polynomial $P_{\sigma}$ and by $\alpha_{r+1}, \ldots, \alpha_{r+s}, \overline{\alpha_{r+1}}, \ldots, \overline{\alpha_{r+s}}$ its $2 s$ complex roots. With this notation, we have $2 s+r=d$. Let $\mathbb{K}_{k}$ be the complete field containing $\alpha_{k}$, that is to say $\mathbb{R}$ if $1 \leq k \leq r$ and $\mathbb{C}$ if $k>r$.

Any right eigenvector $\mathbf{u}$ for the eigenvalue $\alpha$ satisfies $\left(\mathbf{M}_{\sigma}-\alpha I\right) \mathbf{u}=0$. This system has solutions in the field $\mathbb{Q}(\alpha)$, so that there exists a vector $\mathbf{u}_{\mathbf{1}} \neq 0$ such that

$$
\mathbf{M}_{\sigma} \mathbf{u}_{\mathbf{1}}=\alpha \mathbf{u}_{\mathbf{1}} \quad \text { with } \quad \mathbf{u}_{\mathbf{1}} \in \mathbb{Q}(\alpha)^{d} .
$$

Basic linear algebra tells us that $\mathbf{u}_{\mathbf{1}}$ is orthogonal to each left eigenvector of $\mathbf{M}_{\sigma}$ for an eigenvalue different from $\alpha$. Since the matrix $\mathbf{M}_{\sigma}$ is diagonalizable, this implies that $\mathbf{u}_{\mathbf{1}}$ is not orthogonal to any left eigenvector of $\mathbf{M}_{\sigma}$ for $\alpha$. Thus, if $\langle\mathbf{u}, \mathbf{v}\rangle={ }^{t} \overline{\mathbf{u}} \mathbf{v}$ denote the scalar product in $\mathbb{C}^{d}$, there exists a vector $\mathbf{v}_{\mathbf{1}} \neq 0$ such that

$$
{ }^{t} \mathbf{v}_{\mathbf{1}} \mathbf{M}_{\sigma}=\alpha^{t} \mathbf{v}_{\mathbf{1}} \quad \text { and } \quad\left\langle\mathbf{u}_{\mathbf{1}}, \mathbf{v}_{\mathbf{1}}\right\rangle=1 \quad \text { with } \quad \mathbf{v}_{\mathbf{1}} \in \mathbb{Q}(\alpha)^{d} .
$$

We obtain in the next lemma two bases of right and left eigenvectors of $\mathbf{M}_{\sigma}$ by replacing $\alpha$ with each eigenvalue $\alpha_{i}$ in the coordinates of $\mathbf{u}_{\mathbf{1}}$ and $\mathbf{v}_{\mathbf{1}}$. We recall that the canonical morphism from $\mathbb{Q}(\alpha)$ onto $\mathbb{Q}\left(\alpha_{k}\right)$ is the field homomorphism which sends $\alpha$ on $\alpha_{k}$ and is equal to the identity map on $\mathbb{Q}$.

Lemma 2.1. Let $\alpha_{k}$ be an eigenvalue of $\mathbf{M}_{\sigma}$ and let $\sigma_{k}$ be the canonical morphism from $\mathbb{Q}(\alpha)$ onto $\mathbb{Q}\left(\alpha_{k}\right)$, extended to $\mathbb{Q}(\alpha)^{d}$.

Let $\mathbf{u}_{\mathbf{k}}$ and $\mathbf{v}_{\mathbf{k}}$ be the vectors defined by

$$
\mathbf{u}_{\mathbf{k}}=\sigma_{k}\left(\mathbf{u}_{\mathbf{1}}\right) \text { and } \quad \mathbf{v}_{\mathbf{k}}=\sigma_{k}\left(\mathbf{v}_{\mathbf{1}}\right) \text {. }
$$

Then the family $\left\{\mathbf{u}_{\mathbf{1}}, \ldots, \mathbf{u}_{\mathbf{r}}, \mathbf{u}_{\mathbf{r}+\mathbf{1}}, \overline{\mathbf{u}_{\mathbf{r}+\mathbf{1}}}, \ldots, \mathbf{u}_{\mathbf{r}+\mathbf{s}}, \overline{\mathbf{u}_{\mathbf{r}+\mathbf{s}}}\right\}$ is a basis of $\mathbb{C}^{d}$ consisting of right eigenvectors of $\mathbf{M}_{\sigma}$, while $\left\{\mathbf{v}_{\mathbf{1}}, \ldots, \mathbf{v}_{\mathbf{r}}, \overline{\mathbf{v}_{\mathbf{r}+\mathbf{1}}}, \mathbf{v}_{\mathbf{r}+\mathbf{1}}, \ldots, \overline{\mathbf{v}_{\mathbf{r}+\mathbf{s}}}, \mathbf{v}_{\mathbf{r}+\mathbf{s}}\right\}$ is a basis of left eigenvectors

$$
\mathbf{M}_{\sigma} \mathbf{u}_{\mathbf{k}}=\alpha_{k} \mathbf{u}_{\mathbf{k}} \quad \text { and } \quad{ }^{t} \mathbf{v}_{\mathbf{k}} \mathbf{M}_{\sigma}=\alpha_{k}{ }^{t} \mathbf{v}_{\mathbf{k}} \quad \text { with } \quad \mathbf{u}_{\mathbf{k}}, \mathbf{v}_{\mathbf{k}} \in \mathbb{Q}\left(\alpha_{k}\right)^{d} .
$$

If we apply the canonical morphisms to the normalization formula $\left\langle\mathbf{u}_{\mathbf{1}}, \mathbf{v}_{\mathbf{1}}\right\rangle=1$ and remember that left and right eigenvectors for different eigenvalues are orthogonal, we get the following formulas, which mean that the two bases are dual bases:

$$
\begin{aligned}
& \text { if } k=1 \ldots r \\
& \text { if } k=r+1 \ldots r+s
\end{aligned}\left\{\begin{array}{l}
\left\langle\mathbf{u}_{\mathbf{k}}, \mathbf{v}_{\mathbf{k}}\right\rangle=1, \\
\left\langle\mathbf{u}_{\mathbf{j}}, \mathbf{v}_{\mathbf{k}}\right\rangle\left\langle\overline{\mathbf{u}_{\mathbf{j}}}, \mathbf{v}_{\mathbf{k}}\right\rangle=0 \text { for } j \neq k, \\
\left\langle\overline{\mathbf{u}_{\mathbf{k}}}, \mathbf{v}_{\mathbf{k}}\right\rangle=1, \\
\left\langle\mathbf{u}_{\mathbf{k}}, \mathbf{v}_{\mathbf{k}}\right\rangle=0, \\
\left\langle\mathbf{u}_{\mathbf{j}}, \mathbf{v}_{\mathbf{k}}\right\rangle=\left\langle\overline{\mathbf{u}_{\mathbf{j}}}, \mathbf{v}_{\mathbf{k}}\right\rangle=0 \text { for } j \neq k .
\end{array}\right.
$$

Let $R_{c}=\left\{\alpha_{2}, \ldots, \alpha_{r}, \alpha_{r+1}, \ldots, \alpha_{s+r}\right\}$ be the set of eigenvalues of modulus smaller than 1 , in which there is no pair of complex conjugates. For every eigenvalue $\alpha_{k}$ in $R_{c}$, let $\delta_{k}$ be the map which sends any finite word $W$ of $\mathcal{A}^{*}$ to the scalar 


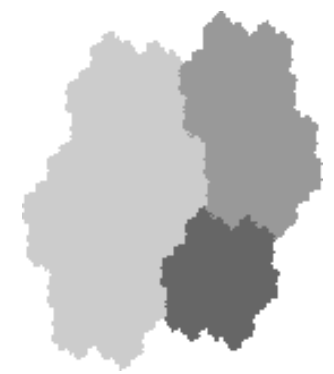

Figure 1. The Rauzy fractal

product of $\mathbf{v}_{\mathbf{k}}$ by the vector $\mathbf{l}(W)=\left(|W|_{i}\right)_{i=1, \ldots, d}$.

$$
\begin{aligned}
\delta_{k}: \mathcal{A}^{*} & \rightarrow \mathbb{K}_{k} \\
W & \mapsto\left\langle\mathbf{l}(W), \mathbf{v}_{\mathbf{k}}\right\rangle .
\end{aligned}
$$

The following equations are easily deduced from scalar product bilinearity, the fact that $\mathbf{v}_{\mathbf{k}}$ is a left eigenvector of $\mathbf{M}_{\sigma}$, and from formula (1.1).

$$
\begin{array}{lrl}
\forall j \in \mathcal{A} & \delta_{k}(j) & =\left(\mathbf{v}_{\mathbf{k}}\right)_{j}, \\
\forall W_{1}, W_{2} \in \mathcal{A}^{*} & \delta_{k}\left(W_{1} W_{2}\right) & =\delta_{k}\left(W_{1}\right)+\delta_{k}\left(W_{2}\right), \\
\forall W \in \mathcal{A}^{*} & \delta_{k}(\sigma(W)) & =\alpha_{k} \delta_{k}(W) .
\end{array}
$$

Set the digit map:

$$
\begin{aligned}
\delta: \mathcal{A}^{*} & \rightarrow \mathbb{R}^{r-1} \times \mathbb{C}^{s} \\
W & \mapsto\left(\delta_{k}(W)\right)_{2 \leq k \leq r+s} .
\end{aligned}
$$

The map $\delta$ is a homomorphism on $\mathcal{A}^{*}$ with respect to concatenation

$$
\forall W_{1}, W_{2} \in \mathcal{A}^{*} \quad \delta\left(W_{1} W_{2}\right)=\delta\left(W_{1}\right)+\delta\left(W_{2}\right) .
$$

Moreover, if $\mathbf{M}_{\text {diag }} \in \mathcal{M}_{s+r-1}(\mathbb{C})$ denotes the diagonal matrix of size $s+r-1$ containing the contracting eigenvalues in $R_{c}, \mathbf{M}_{\text {diag }}$ and $\sigma$ satisfy a commutation relation:

$$
\forall W \in \mathcal{A}^{*}, \quad \delta(\sigma(W))=\mathbf{M}_{\text {diag }} \delta(W) \quad \mathbf{M}_{\text {diag }}=\left(\begin{array}{ccc}
\alpha_{2} & & (0) \\
& \ddots & \\
(0) & & \alpha_{r+s}
\end{array}\right) .
$$

The Rauzy fractal (Figure 1) is obtained in 27] as the closure of the points $\delta(W)$, over all the prefixes $W$ of a fixed point of the substitution. With the same definition, one can build a compact set for every substitution of Pisot type (see [2, 31]). Some properties of these compact sets are proved in 15, 24, 31. The aim of Section 2.2 is to extend all $\Omega$ onto this compact set, and the map defined on the orbit of the periodic points of $\Omega$ to obtain the compact set.

2.2. Representation of the substitutive system in a geometrical space. Proposition 1.4 implies that Theorem [1.7] is satisfied by every substitution of Pisot type: the prefix-suffix development $\Gamma$ is well defined on $\Omega$. In the next lemma, thanks to this development, we define on $\Omega$ a map which is equivalent to gathering in a vector all the geometric representations of C. Holton and L. Zamboni [15]. 
Lemma 2.2. Let $w$ be a word in $\Omega$ and let $\Gamma(w)=\left(p_{i}, a_{i}, s_{i}\right)_{i \geq 0}$ be its prefix-suffix development. The sequence of points $\delta\left(\sigma^{n}\left(p_{n}\right) \ldots \sigma^{0}\left(p_{0}\right)\right)$ has a limit in the space $\mathbb{R}^{r-1} \times \mathbb{C}^{s}$ as $n$ tends to infinity. The following map $\varphi$, called the representation map of $\Omega$ in the space, is well-defined from $\Omega$ on $\mathbb{R}^{r-1} \times \mathbb{C}^{s}$ and is continuous.

$$
\begin{aligned}
\varphi(w) & =\lim _{n \rightarrow+\infty} \delta\left(\sigma^{n}\left(p_{n}\right) \ldots \sigma^{0}\left(p_{0}\right)\right) \\
& =\sum_{i \geq 0} \mathbf{M}_{\text {diag }}^{i} \delta\left(p_{i}\right)=\left(\begin{array}{c}
\sum_{i \geq 0} \delta_{2}\left(p_{i}\right) \alpha_{2}{ }^{i} \\
\vdots \\
\sum_{i \geq 0} \delta_{r+s}\left(p_{i}\right) \alpha_{r+s}{ }^{i}
\end{array}\right) .
\end{aligned}
$$

Proof. The three equalities are deduced from formula (2.4) which implies

$$
\delta\left(\sigma^{n}\left(p_{n}\right) \ldots \sigma^{0}\left(p_{0}\right)\right)=\sum_{i=0}^{n} \mathbf{M}_{d i a g}^{i} \delta\left(p_{i}\right) .
$$

The map $\varphi$ is well-defined and continuous because it is the limit of a power series which is normally convergent, since the number of vectors $\delta\left(p_{j}\right)$ is finite and the norm of $\mathbf{M}_{\text {diag }}$ smaller than 1: $\left\|\mathbf{M}_{\text {diag }}\right\|=\max \left\{|\beta|, \beta \in R_{c}\right\}<1$.

The way the shift map $S$ and the substitution $\sigma$ operate on the prefix-suffix development map (Lemma 1.9) permits us to express how $S$ and $\sigma$ operate in the space $\mathbb{R}^{r-1} \times \mathbb{C}^{s}$ via the map $\varphi$ :

Proposition 2.3. For every $w$ in $\Omega$, if $w_{0}$ is the 0 th coordinate of $w$, we have

$$
\begin{aligned}
& \varphi(S w)=\varphi(w)+\delta\left(w_{0}\right), \\
& \varphi(\sigma w)=\mathbf{M}_{\text {diag }} \varphi(w) .
\end{aligned}
$$

Proof. Let $\Gamma(w)=\left(p_{i}, a_{i}, s_{i}\right)_{i \geq 0}, \Gamma(S w)=\left(q_{i}, b_{i}, t_{i}\right)_{i \geq 0}$ and $\Gamma(\sigma w)=\left(r_{i}, c_{i}, u_{i}\right)_{i \geq 0}$, be the prefix-suffix developments of $w, S w$ and $\sigma w$ respectively.

According to Lemma 1.9, if $S w$ is not a periodic point of $\sigma$, we have

$$
\exists n_{0}, \quad \forall n \geq n_{0} \quad \sigma^{n}\left(p_{n}\right) \ldots \sigma^{0}\left(p_{0}\right) w_{0}=\sigma^{n}\left(q_{n}\right) \ldots \sigma^{0}\left(q_{0}\right) .
$$

This is enough to prove the equality (2.5) if $S w$ is not periodic :

$$
\begin{aligned}
\varphi(S w) & =\lim _{n \rightarrow+\infty} \delta\left(\sigma^{n}\left(q_{n}\right) \ldots \sigma^{0}\left(q_{0}\right)\right) \\
& =\lim _{n \rightarrow+\infty} \delta\left(\sigma^{n}\left(p_{n}\right) \ldots \sigma^{0}\left(p_{0}\right) w_{0}\right) \\
& =\lim _{n \rightarrow+\infty} \delta\left(\sigma^{n}\left(p_{n}\right) \ldots \sigma^{0}\left(p_{0}\right)\right)+\delta\left(w_{0}\right) \\
& =\varphi(w)+\delta\left(w_{0}\right) .
\end{aligned}
$$

If $S w$ is a periodic point of $\sigma$, from Lemma 1.9, the development of $S w$ has empty prefixes, which imply $\varphi(S w)=0$. Moreover, the sequence $\left(p_{i}\right)_{i \geq 0}$ is periodic of period $l$. Thus, we get for every integer $n$ and every $i<l$,

$$
\sigma^{i+n l}\left(p_{i+n l}\right) \ldots p_{0} w_{0}=\sigma^{n l}\left(\sigma^{i}\left(p_{i}\right) \ldots p_{0} w_{0}\right)
$$

which implies

$$
\delta\left(\sigma^{i+n l}\left(p_{i+n l}\right) \ldots p_{0} w_{0}\right)=\mathbf{M}_{\text {diag }}{ }^{n l} \delta\left(\sigma^{i}\left(p_{i}\right) \ldots p_{0} w_{0}\right) .
$$



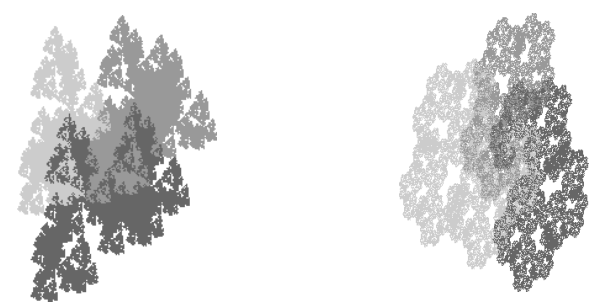

Figure 2. Fractals generated by $132,21323,321323132$ and $12,3,31$

Since the number of vectors $\delta\left(\sigma^{i}\left(p_{i}\right) \ldots p_{0} w_{0}\right)$ is finite and $\mathbf{M}_{\text {diag }}$ is a contracting linear map, we conclude:

$$
\begin{aligned}
\varphi(w) & =\lim _{n \rightarrow+\infty} \delta\left(\sigma^{n}\left(p_{n}\right) \ldots p_{0}\right) \\
& =\lim _{n \rightarrow+\infty} \delta\left(\sigma^{n}\left(p_{n}\right) \ldots p_{0} w_{0}\right)-\delta\left(w_{0}\right) \\
& =\lim _{n \rightarrow+\infty} \mathbf{M}_{\text {diag }}{ }^{n l} \delta\left(\sigma^{i}\left(p_{i}\right) \ldots p_{0} w_{0}\right)-\delta\left(w_{0}\right) \\
& =0-\delta\left(w_{0}\right)=\varphi(S w)-\delta\left(w_{0}\right) .
\end{aligned}
$$

Finally, the definition of $r_{i}=p_{i-1}$ for $i \geq 1$ leads to formula (2.6):

$$
\begin{aligned}
\varphi(\sigma w) & =\mathbf{M}_{\text {diag }}^{0} \delta(\varepsilon)+\sum_{j \geq 1} \mathbf{M}_{\text {diag }}^{j} \delta\left(p_{j-1}\right) \\
& =\mathbf{M}_{\text {diag }} \varphi(w) .
\end{aligned}
$$
2

Set $\mathcal{F}=\varphi(\Omega)$ and $\mathcal{F}_{i}=\varphi([i])$ for each letter $i$. Two examples are given in Figure

Relations (2.5) and (2.6) suggest that we could define an exchange of domains $\mathcal{T}$ on $\mathcal{F}$ which satisfies a conjugacy relation with the shift $S$. As the map exchange would be different on each image cylinder $\varphi[i]=\mathcal{F}_{i}$, such a definition is possible only if the images of cylinders are completely disjoint, which is not the case even for Rauzy substitution (see [24]). Nevertheless, for Rauzy substitution, the domains $\mathcal{F}_{i}$ are disjoint up to a set of measure zero, and it is possible to define an exchange of domains in $\mathcal{F}$ which is measurably conjugate with the shift on $\Omega$. We will prove in Section 4 that this is more generally true when the substitution verifies the combinatorial condition of coincidences.

Another way to define a factor of $(\Omega, S)$ is to quotient the space in such a way that the projections of the vectors $\delta(k)$ defining the exchange of domains are equal. This is done in Section 3, using the geometrical interpretation of the map $\varphi$ given in the next subsection.

2.3. Geometrical interpretation: equivalent representation in $\mathbb{R}^{d-1}$. In the next lemma, we modify the basis of $\mathbb{C}^{d}$ consisting of right eigenvectors of $\mathbf{M}_{\sigma}$ and obtain a basis in $\mathbb{R}^{d}$.

Lemma 2.4. The following family $\mathcal{B}$ of vectors is a basis of $\mathbb{R}^{d}$ :

$$
\mathcal{B}=\left\{\mathbf{u}_{\mathbf{1}}, \ldots, \mathbf{u}_{\mathbf{r}}, \operatorname{Re} \mathbf{u}_{\mathbf{r}+\mathbf{1}}, \operatorname{Im} \mathbf{u}_{\mathbf{r}+\mathbf{1}}, \ldots, \operatorname{Re} \mathbf{u}_{\mathbf{r}+\mathbf{s}}, \operatorname{Im} \mathbf{u}_{\mathbf{r}+\mathbf{s}}\right\} .
$$


Proof. Consider a real linear combination of the vectors equal to zero:

$$
\sum_{k=1}^{r} a_{k} \mathbf{u}_{\mathbf{k}}+\sum_{k=r+1}^{r+s}\left(b_{k} \operatorname{Re} \mathbf{u}_{\mathbf{k}}+c_{k} \operatorname{Im} \mathbf{u}_{\mathbf{k}}\right)=0
$$

This can be rewritten in $\mathbb{C}^{d}$ :

$$
\sum_{k=1}^{r} a_{k} \mathbf{u}_{\mathbf{k}}+\sum_{k=r+1}^{r+s}\left(\left(\frac{b_{k}-i c_{k}}{2}\right) \mathbf{u}_{\mathbf{k}}+\left(\frac{b_{k}+i c_{k}}{2}\right) \mathbf{u}_{\mathbf{k}+\mathbf{s}}\right)=0 .
$$

Linear independence of the vectors $\mathbf{u}_{\mathbf{i}}$ in $\mathbb{C}^{d}$ (Lemma 2.1) implies that $a_{k}=$ $\frac{b_{k}-i c_{k}}{2}=0$ for all $k$, that is $a_{k}=b_{k}=c_{k}=0$ for all $k$.

We call a contracting hyperplane of $\mathbf{M}_{\sigma}$ the hyperplane of $\mathbb{R}^{d}$ generated by the vectors

$$
\mathbf{u}_{\mathbf{2}}, \ldots, \mathbf{u}_{\mathbf{r}}, \operatorname{Re} \mathbf{u}_{\mathbf{r}+\mathbf{1}}, \operatorname{Im} \mathbf{u}_{\mathbf{r}+\mathbf{1}}, \ldots, \operatorname{Re} \mathbf{u}_{\mathbf{r}+\mathbf{s}}, \operatorname{Im} \mathbf{u}_{\mathbf{r}+\mathbf{s}}
$$

The space $\mathbb{R}^{r-1} \times \mathbb{C}^{s}$ embeds onto this hyperplane:

Corollary 2.5. The following linear map $p_{\mathcal{H}}: \mathbb{R}^{r-1} \times \mathbb{C}^{s} \rightarrow \mathbb{R}^{d}$ is one-to-one from $\mathbb{R}^{r-1} \times \mathbb{C}^{s}$ onto the contracting hyperplane of $\mathbf{M}_{\sigma}$.

$$
p_{\mathcal{H}}\left(\lambda_{2}, \ldots, \lambda_{r+s}\right)=\sum_{k=2}^{r} \lambda_{k} \mathbf{u}_{\mathbf{k}}+\sum_{k=r+1}^{r+s}\left(2 \operatorname{Re}\left(\lambda_{k}\right) \operatorname{Re} \mathbf{u}_{\mathbf{k}}-2 \operatorname{Im}\left(\lambda_{k}\right) \operatorname{Im} \mathbf{u}_{\mathbf{k}}\right) .
$$

The next lemma means that applying the map $\delta$ to a finite word $W$ is equivalent to projecting the vector $\mathbf{l}(W)$ onto the contracting hyperplane of the matrix $\mathbf{M}_{\sigma}$ parallel to the expanding direction of $\mathbf{M}_{\sigma}$.

Lemma 2.6. If $q_{\mathcal{H}}$ denote the linear projection of $\mathbb{R}^{d}$ onto the contracting hyperplane of $\mathbf{M}_{\sigma}$ parallel to the expanding direction of $\mathbf{M}_{\sigma}$, then

$$
\forall W \in \mathcal{A}^{*} \quad p_{\mathcal{H}} \delta(W)=q_{\mathcal{H}} \mathbf{l}(W) .
$$

Proof. By definition of $p_{\mathcal{H}}$ and $\delta$, we have

$$
p_{\mathcal{H}} \delta(W)=\sum_{k=2}^{r}\left\langle\mathbf{l}(W), \mathbf{v}_{\mathbf{k}}\right\rangle \mathbf{u}_{\mathbf{k}}+\sum_{k=r+1}^{r+s} 2 \operatorname{Re}\left\langle\mathbf{l}(W), \mathbf{v}_{\mathbf{k}}\right\rangle \operatorname{Re} \mathbf{u}_{\mathbf{k}}-2 \operatorname{Im}\left\langle\mathbf{l}(W), \mathbf{v}_{\mathbf{k}}\right\rangle \operatorname{Im} \mathbf{u}_{\mathbf{k}} .
$$

Write the vector $\mathbf{l}(W)$ in the basis $\mathcal{B}$ of the contracting hyperplane $\mathcal{H}$ :

$$
\begin{aligned}
\mathbf{l}(W) & =\sum_{k=1}^{r} \lambda_{k} \mathbf{u}_{\mathbf{k}}+\sum_{k=r+1}^{r+s}\left(\mu_{k} \operatorname{Re} \mathbf{u}_{\mathbf{k}}+\nu_{k} \operatorname{Im} \mathbf{u}_{\mathbf{k}}\right) \\
& =\sum_{k=1}^{r} \lambda_{k} \mathbf{u}_{\mathbf{k}}+\sum_{k=r+1}^{r+s}\left(\mu_{k}-i \nu_{k}\right) / 2 \mathbf{u}_{\mathbf{k}}+\left(\mu_{k}+i \nu_{k}\right) / 2 \overline{\mathbf{u}_{\mathbf{k}}}
\end{aligned}
$$

The fact that the $\mathbf{u}_{\mathbf{k}}$ are the dual basis of the $\mathbf{v}_{\mathbf{k}}$ (formula (2.1)) implies that

$$
\begin{aligned}
k=1 \ldots r & \left\langle\mathbf{l}(W), \mathbf{v}_{\mathbf{k}}\right\rangle=\lambda_{k}, \\
k=r+1 \ldots r+s & \left\langle\mathbf{l}(W), \mathbf{v}_{\mathbf{k}}\right\rangle=\left(\mu_{k}-i \nu_{k}\right) / 2 .
\end{aligned}
$$

So that we get

$$
\mathbf{l}(W)=\left\langle\mathbf{l}(W), \mathbf{v}_{\mathbf{1}}\right\rangle \mathbf{u}_{\mathbf{1}}+p_{\mathcal{H}} \delta(W)
$$




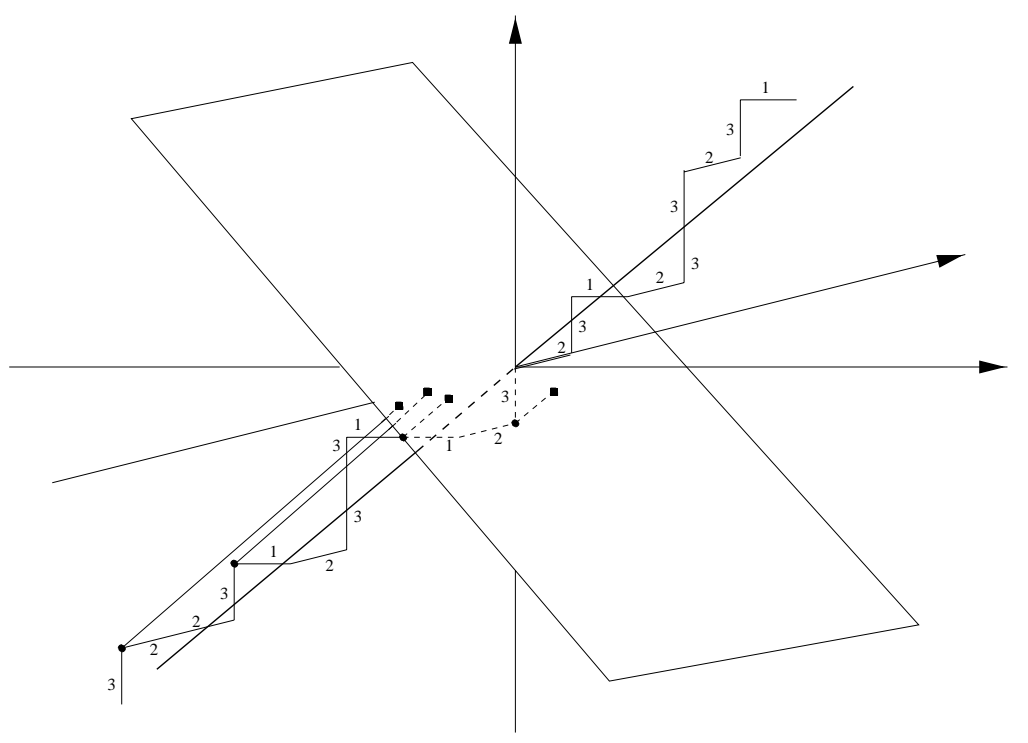

Figure 3. Projection on the hyperplane

Since $q_{\mathcal{H}}$ sends $\mathbf{u}_{\mathbf{1}}$ to zero and leaves the contracting hyperplane of $\mathbf{M}_{\sigma}$ unchanged, we conclude that

$$
q_{\mathcal{H}} \mathbf{l}(W)=\left\langle\mathbf{l}(W), \mathbf{v}_{\mathbf{1}}\right\rangle q_{\mathcal{H}}\left(\mathbf{u}_{\mathbf{1}}\right)+q_{\mathcal{H}} p_{\mathcal{H}} \delta(W)=p_{\mathcal{H}} \delta(W) .
$$

Consequently, one way to construct the image $\varphi(\Omega)$ is to consider an infinite word $w$ in $\Omega$, embed it as a broken line in the space $\mathbb{R}^{d}$ (replacing each letter $i$ by the $i$ th canonical vector), and then project each "vertex" of the broken line on the contracting hyperplane of $\mathbf{M}_{\sigma}$ parallel to the expanding direction of $\mathbf{M}_{\sigma}$. The set $\varphi(\Omega)$ is the closure of the projections.

The prefix-suffix development brings us to consider on the broken line the points corresponding to the prefixes $\sigma^{n}\left(p_{n}\right) \ldots \sigma^{0}\left(p_{0}\right)$ : the sequence of projections in the hyperplane of these points has a limit which belongs to $\varphi(\Omega)$, and this limit is $\varphi(w)$ (see Figure 3 ).

\section{Representation of $(\Omega, S)$ on the torus}

To define, in the Pisot case, a commutation relation with a translation, we need to quotient the space so that the projections of the vectors $\delta(k)$ are equal. We prove in this section that the set $\mathcal{L}$ defined by

$$
\mathcal{L}=\left\{\sum_{k=1}^{d} n_{k} \delta(k) ; n_{k} \in \mathbb{Z}, \sum_{k=1}^{d} n_{k}=0\right\}
$$

is a lattice of the $\mathbb{R}$ vector space $\mathbb{R}^{r-1} \times \mathbb{C}^{s}$, that is a discrete subgroup of rank $d-1$, so that the quotient of $\mathbb{R}^{r-1} \times \mathbb{C}^{s}$ modulo this lattice is isomorphic to the torus $\mathbb{T}^{d-1}$. We obtain in that way a representation of $\Omega$ on the torus, which satisfies a commutation relation with a translation. We prove that this translation is minimal.

The main argument in this section is the following proposition: 
Proposition 3.1. The coordinates in the canonical basis of $\mathbb{R}^{d}$ of the expanding right eigenvector $\mathbf{u}_{\mathbf{1}}$ of $\mathbf{M}_{\sigma}$ are rationally independent.

Proof. Let $\left(\lambda_{k}\right)_{1 \leq k \leq d}$ be the coordinates of the vector $\mathbf{u}_{\mathbf{1}}$ in the canonical basis of $\mathbb{R}^{d}$. Consider a rational combination of $\lambda_{k}$ which is equal to zero:

$$
\sum_{j=1}^{d} q_{j} \lambda_{j}=0, \quad\left(q_{j}\right)_{1 \leq j \leq d} \in \mathbb{Q}^{d} .
$$

Since the vector $\mathbf{u}_{\mathbf{1}}$ was defined so that every $\lambda_{j}$ belongs to $\mathbb{Q}(\alpha)$, it is possible to apply the canonical automorphisms $\sigma_{k}: \mathbb{Q}(\alpha) \rightarrow \mathbb{Q}\left(\alpha_{k}\right)$ and $\overline{\sigma_{k}}: \mathbb{Q}(\alpha) \rightarrow \mathbb{Q}\left(\overline{\alpha_{k}}\right)$ to the preceding equality, for every $1 \leq k \leq r+s$, so that we get

$$
\begin{gathered}
k=1 \ldots r+s, \quad \sum_{j=1}^{d} \sigma_{k}\left(q_{j}\right) \sigma_{k}\left(\lambda_{j}\right)=\sum_{j=1}^{d} q_{j} \sigma_{k}\left(\lambda_{j}\right)=0, \\
k=r+1 \ldots r+s, \quad \sum_{j=1}^{d} q_{j} \overline{\sigma_{k}}\left(\lambda_{j}\right)=0 .
\end{gathered}
$$

Thus, the vector $\left(q_{j}\right)_{1 \leq j \leq d}$ is orthogonal in $\mathbb{C}^{d}$ to the vectors $\left(\sigma_{k}\left(\lambda_{j}\right)\right)_{1 \leq j \leq d}$ and $\left(\overline{\sigma_{k}}\left(\lambda_{j}\right)\right)_{1 \leq j \leq d}$, which are by construction equal to $\mathbf{u}_{\mathbf{k}}$ and $\overline{\mathbf{u}_{\mathbf{k}}}$. This means that the vector $\left(q_{j}\right)_{1 \leq j \leq d}$ is orthogonal to a full basis of the space $\mathbb{C}^{d}$, and must be zero.

By definition, the set $\mathcal{L}$ is discrete and generated by the $d-1$ vectors $(\delta(k)-$ $\delta(1))_{2 \leq k \leq d}$ in $\mathbb{R}^{r-1} \times \mathbb{C}^{s}$. Thus, this set is a lattice if and only if the vectors $(\delta(k)-\delta(1))_{2 \leq k \leq d}$ are linearly independent.

Lemma 3.2. The $d-1$ vectors $(\delta(k)-\delta(1))_{2 \leq k \leq d}$ are $\mathbb{R}$-linearly independent in $\mathbb{R}^{r-1} \times \mathbb{C}^{s}$, so that they form a basis of this $\mathbb{R}$ vector space.

Proof. Since the embedding $p_{\mathcal{H}}$ is linear and one-to-one, the vectors $\delta(k)-\delta(1)$ are free if and only if the vectors $p_{\mathcal{H}} \delta(k)-p_{\mathcal{H}} \delta(1)$ are free in $\mathbb{R}^{d}$. Consider a linear combination of those vectors which is equal to zero:

$$
\sum_{k=2}^{d} \lambda_{k}\left(p_{\mathcal{H}} \delta(k)-p_{\mathcal{H}} \delta(1)\right)=0 .
$$

Denote by $\mathbf{e}_{\mathbf{1}}, \ldots \mathbf{e}_{\mathbf{d}}$ the canonical basis of $\mathbb{R}^{d}$. Lemma 2.6 implies

$$
q_{\mathcal{H}}\left(\sum_{k=2}^{d} \lambda_{k}\left(\mathbf{e}_{\mathbf{k}}-\mathbf{e}_{\mathbf{1}}\right)\right)=0 .
$$

Since $q_{\mathcal{H}}$ is a projection parallel to $\mathbf{u}_{\mathbf{1}}$, its kernel is generated by $\mathbf{u}_{\mathbf{1}}$ and there exists a real $\nu$ such that

$$
-\left(\sum_{k=2}^{d} \lambda_{k}\right) \mathbf{e}_{\mathbf{1}}+\sum_{k=2}^{d} \lambda_{k} \mathbf{e}_{\mathbf{k}}=\nu \mathbf{u}_{\mathbf{1}} .
$$

If $\nu$ is different from zero, the coordinates of $\mathbf{u}_{\mathbf{1}}$ in the canonical basis are rationally dependent, which is impossible (Proposition [3.1). Thus, $\nu$ is zero and the independence of the vectors $\mathbf{e}_{\mathbf{k}}$ implies that all the $\lambda_{k}$ are also equal to zero. 
Corollary 3.3. The following map $i_{\mathcal{L}}$ is an isomorphism from the $(d-1)$-torus $\mathbb{T}^{d-1}$ onto the quotient $\left(\mathbb{R}^{r-1} \times \mathbb{C}^{s}\right) / \mathcal{L}$.

$$
\begin{aligned}
i_{\mathcal{L}}: \quad \mathbb{T}^{d-1}=\mathbb{R}^{d-1} / \mathbb{Z}^{d-1} & \rightarrow\left(\mathbb{R}^{r-1} \times \mathbb{C}^{s}\right) / \mathcal{L} \\
\left(\lambda_{2}, \ldots, \lambda_{d}\right) \bmod \mathbb{Z}^{d-1} & \mapsto \sum_{k=2}^{d} \lambda_{k}(\delta(k)-\delta(1)) \bmod \mathcal{L}
\end{aligned}
$$

We can define the geometric representation map on the torus of the dynamical system:

Corollary 3.4. If $\varphi$ is the representation map of $\Omega$ in $\mathbb{R}^{r-1} \times \mathbb{C}^{s}$ (Lemma 2.2), the following map is well defined and continuous. We call it the representation map of $\Omega$ on the torus.

$$
\begin{aligned}
\varphi_{\mathcal{L}}: \quad \Omega & \rightarrow\left(\mathbb{R}^{r-1} \times \mathbb{C}^{s}\right) / \mathcal{L} \simeq \mathbb{T}^{d-1} \\
w & \mapsto \varphi(w) \bmod \mathcal{L} .
\end{aligned}
$$

For every $w \in \Omega$, the following equality is satisfied in $\left(\mathbb{R}^{r-1} \times \mathbb{C}^{s}\right) / \mathcal{L}$ :

$$
\varphi_{\mathcal{L}}(S w)=\varphi_{\mathcal{L}}(w)+\delta(1) .
$$

Thus, the diagram commutes:

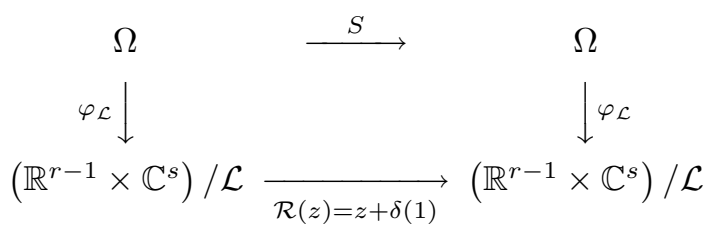

Corollary 3.3 means that the dynamical system $\left(\left(\mathbb{R}^{r-1} \times \mathbb{C}^{s}\right) / \mathcal{L}, \mathcal{R}\right)$ is conjugate to the addition of $i_{\mathcal{L}}{ }^{-1}(\delta(1))$ on the torus $\mathbb{T}^{d-1}$, so that the minimality of one of these dynamical systems is equivalent to the minimality of the other. However, minimal translations on the torus are characterized by the following property.

Proposition 3.5 (see [20]). The translation of vector $\mathbf{t}=\left(t_{1}, \ldots, t_{n}\right) \in \mathbb{R}^{n}$ over the $n$-dimensional torus $\mathbb{T}^{n}$ is minimal if and only if the numbers $t_{1}, \ldots, t_{n}$ and 1 are rationally independent.

Thus, the map $\mathcal{R}$ is minimal if and only if the coordinates of $i_{\mathcal{L}}{ }^{-1}(\delta(1))$ in the canonical basis are rationally independent from 1 . We note that this is not equivalent, but in fact stronger than to prove that the vector $i_{\mathcal{L}}{ }^{-1}(\delta(1))$ is rationally independent of the vectors generating the lattice $\mathbb{Z}^{d-1}$, that is the canonical basis.

Proposition 3.6. The number 1 is rationally independent from the coordinates of $\delta(1)$ in the family $(\delta(k)-\delta(1))_{2 \leq k \leq d}$.

Proof. Denote $\lambda_{k}$ the coordinates of $\delta(1)$ in the basis $(\delta(k)-\delta(1))_{2 \leq k \leq d}$ of $\mathbb{R}^{r-1} \times \mathbb{C}^{s}$ :

$$
\delta(1)=\sum_{k=2}^{d} \lambda_{k}(\delta(k)-\delta(1)) .
$$

If $\left\{\mathbf{e}_{\mathbf{1}}, \ldots, \mathbf{e}_{\mathbf{d}}\right\}$ denote the canonical basis of $\mathbb{R}^{d}$, applying the embedding $p_{\mathcal{H}}$ to this equality and using Lemma 2.6 implies

$$
q_{\mathcal{H}}\left(\left(1+\sum_{k=2}^{d} \lambda_{k}\right) \mathbf{e}_{\mathbf{1}}-\sum_{k=2}^{d} \lambda_{k} \mathbf{e}_{\mathbf{k}}\right)=0 .
$$


But the kernel of $q_{\mathcal{H}}$ is the expanding direction of $\mathbf{M}_{\sigma}$, so that their exists a real $\nu$ with

$$
\nu \mathbf{u}_{\mathbf{1}}=\left(1+\sum_{k=2}^{d} \lambda_{k}\right) \mathbf{e}_{\mathbf{1}}-\sum_{k=2}^{d} \lambda_{k} \mathbf{e}_{\mathbf{k}} .
$$

The number $\nu$ cannot be zero, otherwise every $\lambda_{k}$ would be zero and the preceding equality would become $\mathbf{e}_{\mathbf{1}}=0$. If the $\lambda_{k}$ and 1 are rationally dependent, then the first coordinate of $\mathbf{u}_{\mathbf{1}}$ is a rational combination of $\lambda_{k}$, which are the other coordinates of $\mathbf{u}_{\mathbf{1}}$ in the canonical basis. Proposition 3.1 tells us that this is impossible.

As $i_{\mathcal{L}}$ is an isomorphism, we get that the addition of $i_{\mathcal{L}}{ }^{-1}(\delta(1))$ on the torus $\mathbb{T}^{d-1}$ is minimal, so that:

Corollary 3.7. The dynamical system $\left(\left(\mathbb{R}^{r-1} \times \mathbb{C}^{s}\right) / \mathcal{L}, \mathcal{R}\right)$ is minimal.

We deduce from this that $\varphi_{\mathcal{L}}$ is onto.

Theorem 3.8. Any dynamical system $(\Omega, S)$ generated by a substitution of Pisot type on d letters admits as a topological factor a minimal translation on the torus $\mathbb{T}^{d-1}$.

Indeed, the map $i_{\mathcal{L}}{ }^{-1} \circ \varphi_{\mathcal{L}}$ is continuous and onto, and realizes a semi-conjugation between the shift map $S$ on $\Omega$ and a minimal translation on $\mathbb{T}^{d-1}$.

Proof. The representation map $\varphi_{\mathcal{L}}$ is such that for all $w \in \Omega$

$$
\varphi_{\mathcal{L}}(S(w))=\mathcal{R}\left(\varphi_{\mathcal{L}}(w)\right)
$$

The map $\mathcal{R}$ in minimal so that if $w$ is a point of $\Omega$, the orbit of $\varphi_{\mathcal{L}}(w)$ under $\mathcal{R}$ is dense in $\left(\mathbb{R}^{r-1} \times \mathbb{C}^{s}\right) / \mathcal{L}$. Thus we have

$$
\begin{aligned}
\left(\mathbb{R}^{r-1} \times \mathbb{C}^{s}\right) / \mathcal{L} & =\overline{\left\{\varphi_{\mathcal{L}}(w)+n \delta(1), n \in \mathbb{Z}\right\}}=\overline{\left\{\varphi_{\mathcal{L}}\left(S^{n} w\right), n \in \mathbb{Z}\right\}} \\
& =\varphi_{\mathcal{L}}\left(\overline{\left\{S^{n} w, n \in \mathbb{Z}\right\}}\right)=\varphi_{\mathcal{L}}(\Omega) .
\end{aligned}
$$

Hence, $\varphi_{\mathcal{L}}$ is onto and $\left(\left(\mathbb{R}^{r-1} \times \mathbb{C}^{s}\right) / \mathcal{L}, \mathcal{R}\right)$ is a factor of $(\Omega, S)$.

This theorem gives some information about the image $\mathcal{F}$ of $\varphi$.

Corollary 3.9. The compact subset $\mathcal{F}=\varphi(\Omega)$ of $\mathbb{R}^{r-1} \times \mathbb{C}^{s}$ has nonempty interior and strictly positive Lebesgue measure.

Proof. The fact that $\varphi_{\mathcal{L}}(\Omega)$ is equal to the full torus $\left(\mathbb{R}^{r-1} \times \mathbb{C}^{s}\right) / \mathcal{L}$ means that $\bigcup_{\mathbf{u} \in \mathcal{L}}(\mathcal{F}+\mathbf{u})$ contains the parallelogram $\left\{\sum_{k=2}^{d} t_{k}(\delta(k)-\delta(1)) \mid 0 \leq t_{k} \leq 1\right\}$, which has nonempty interior since the $(\delta(k)-\delta(1))$ are a basis. As $\mathcal{F}$ is a closed subset of $\mathbb{R}^{r-1} \times \mathbb{C}^{s}$, Baire's theorem implies that there exists a vector $\mathbf{u}$ such that $\mathcal{F}+\mathbf{u}$ has nonempty interior, and so does $\mathcal{F}$. Thus, the measure of this set must be strictly positive.

In what follows, we study more precisely the image $\mathcal{F}$ in the unimodular case. 


\section{Representation With AN EXCHANGE OF DOMAINS}

We suppose from here on that $\sigma$ is of Pisot type and unimodular. We prove in Section 4.1 that the induced measure on $\mathcal{F}$ of the measure on $\Omega$ by the representation map $\varphi$ is proportional to the Lebesgue measure on each cylinder of $\Omega$, which implies that $\varphi$ is one-to-one almost everywhere on cylinders. Furthermore, if the substitution satisfies a certain combinatorial condition, we prove in Section 4.2 that $\varphi$ is one-to-one almost everywhere, which allows us to define in Section 4.3 an exchange of domains on $\mathcal{F}$ which is measure-theoretically conjugate to the shift map on $\Omega$.

4.1. Identification of the induced measure. Recall that by primitivity of $\sigma$, the symbolic dynamical system $\left(\Omega, S, \mathcal{B}, \mu_{\Omega}\right)$ is uniquely ergodic, where the Borel sets are generated by the cylinders $\left[U_{1} . U_{2}\right]$ for $U_{1}, U_{2} \in \mathcal{A}^{*}$, and $\mu_{\Omega}$ is defined on cylinders as follows: $\mu_{\Omega}\left[U_{1} \cdot U_{2}\right]=\lim _{n \rightarrow+\infty}(1 / n)$ card $\left\{i<n ; S^{i}(u)\right.$ begins with $\left.U_{1} U_{2}\right\}$ ( $u$ denotes a periodic point for $\sigma$ ).

The vector of the measure of the cylinders $[i]$ is an eigenvector of $\mathbf{M}_{\sigma}$ corresponding to the Perron-Frobenius eigenvalue. Denote by $\mu_{\mathcal{F}}$ the restriction to $\mathcal{F}=\varphi(\Omega)$ of the Lebesgue measure on $\mathbb{R}^{r-1} \times \mathbb{C}^{s}$.

The following lemma gives a generalization of the work on substitutions on two letters of B. Host in [18].

Lemma 4.1. If $\sigma$ is of Pisot type and unimodular, there exists a nonzero constant $C$ such that for every letter $i$,

$$
\mu_{\mathcal{F}}(\varphi([i]))=C \mu_{\Omega}([i]) .
$$

Proof. The recognizability of $\sigma$ implies that every cylinder $[i]$ can be decomposed in terms of cylinders $[j]$ :

$$
[i]=\bigcup_{\substack{j \in \mathcal{A},(p, i, t) \in \mathcal{P} \\ \sigma(j)=p i t}} S^{|p|} \sigma[j] \quad \text { the union being disjoint. }
$$

Proposition 2.3 then implies

$$
\varphi\left(S^{|p|} \sigma[j]\right)=\delta(p)+\mathbf{M}_{\text {diag }} \varphi[j] .
$$

The map associated to $\mathbf{M}_{\text {diag }}$ is a diagonal mapping. So, for every Borel set $B$ of $\mathbb{R}^{r-1} \times \mathbb{C}^{s}$ :

$$
\begin{aligned}
\mu_{\mathcal{F}}\left(\mathbf{M}_{\text {diag }} B\right) & =\prod_{i=2}^{r}\left|\alpha_{i}\right| \prod_{j=r+1}^{r+s}\left|\alpha_{j}\right|^{2} \mu_{\mathcal{F}}(B) \\
& =\frac{\left|\operatorname{det} \mathbf{M}_{\sigma}\right|}{\alpha} \mu_{\mathcal{F}}(B)=\frac{1}{\alpha} \mu_{\mathcal{F}}(B) .
\end{aligned}
$$

As $\mu_{\mathcal{F}}$ is invariant under translation, we can write

$$
\begin{aligned}
\mu_{\mathcal{F}}(\varphi[i]) & \leq \sum_{\sigma(j)=\text { pit }} \mu_{\mathcal{F}}\left(\delta(p)+\mathbf{M}_{\text {diag }} \varphi[j]\right)=\sum_{\sigma(j)=p i t} \frac{1}{\alpha} \mu_{\mathcal{F}}(\varphi[j]) \\
& =\frac{1}{\alpha} \sum_{j=1}^{d} m_{i, j} \mu_{\mathcal{F}}(\varphi[j])=\frac{1}{\alpha}\left(\mathbf{M}_{\sigma}\left(\mu_{\mathcal{F}}(\varphi[j])\right)_{1 \leq j \leq d}\right)_{i} .
\end{aligned}
$$

The number $\alpha$ is the dominant eigenvalue of the matrix $\mathbf{M}_{\sigma}$, so by PerronFrobenius theorem it follows that the above inequalities are in fact equalities. The 
vector $\left(\mu_{\mathcal{F}}(\varphi[j])\right)_{1 \leq j \leq d}$ is then a dominant eigenvector of $\mathbf{M}_{\sigma}$ and is, since $\alpha$ is a simple eigenvalue, proportional to the eigenvector for $\alpha:\left(\mu_{\Omega}([j])\right)_{1 \leq j \leq d}$. Moreover, the constant of proportionality can not be zero as $\mu_{\mathcal{F}}(\mathcal{F})$ is not zero, by Corollary 3.9.

Corollary 4.2. Let $\sigma$ be a unimodular substitution of Pisot type. If $\sigma(j)=$ pit and $\sigma(k)=q i u$, with $(p, i, t),(q, i, u) \in \mathcal{P}$, then

$$
\mu_{\mathcal{F}}\left(\varphi\left(S^{|p|} \sigma[j]\right) \cap \varphi\left(S^{|q|} \sigma[k]\right)\right)=0 .
$$

Each $\varphi[i]$ is a union, disjoint in measure, of $\varphi\left(S^{|p|} \sigma[j]\right)$ :

$$
\varphi[i]=\bigcup_{\substack{j \in \mathcal{A},(p, i, t) \in \mathcal{P} ; \\ \sigma(j)=p i t}} \varphi\left(S^{|p|} \sigma[j]\right) .
$$

Proof. In the proof of Lemma 4.1, there is equality in measure on every line.

Proposition 4.3. If $\sigma$ is of Pisot type and unimodular, the induced measure on $\mathcal{F}$ of the measure of $\Omega$ under the representation map $\varphi$ is proportional to the Lebesgue measure on each cylinder of $\Omega$ : there exists a constant $C$ such that for every letter $i$ and for every Borel set $B \subset[i]$ of $\Omega$,

$$
\mu_{\mathcal{F}}(\varphi B)=C \mu_{\Omega}(B) .
$$

Proof. From Corollary 1.8 the sequence of partitions is generating in measure:

$$
\Omega=\bigcup_{n, i ; n<\left|\sigma^{k}(i)\right|} S^{n} \sigma^{k}[i]
$$

It suffices to prove the equality of the measures on every subset $S^{n} \sigma^{k}[i]$. However, if $p$ is the prefix of length $n$ of $\sigma^{k}(i)$, we have

$$
\mu_{\mathcal{F}}\left(\varphi\left(S^{n} \sigma^{k}[i]\right)\right)=\mu_{\mathcal{F}}\left(\delta(p)+\mathbf{M}_{\text {diag }}^{k} \varphi[i]\right)=\frac{1}{\alpha^{k}} \mu_{\mathcal{F}}(\varphi[i]) .
$$

Furthermore, $\mu_{\Omega}\left(S^{n} \sigma^{k}[i]\right)=\mu_{\Omega}\left(\sigma^{k}[i]\right)$ from the shift invariance. A similar calculation as in the Lemma 4.1, gives that

$$
\left(\mu_{\Omega}([i])\right)_{1 \leq i \leq d}=\mathbf{M}_{\sigma}\left(\mu_{\Omega}(\sigma[i])\right)_{1 \leq i \leq d}=\mathbf{M}_{\sigma}^{k}\left(\mu_{\Omega}\left(\sigma^{k}[i]\right)\right)_{1 \leq i \leq d} .
$$

Since $\left(\mu_{\Omega}([i])\right)_{1 \leq i \leq d}$ is an eigenvector of $\mathbf{M}_{\sigma}$ we deduce that

$$
\mu_{\Omega}\left(S^{n} \sigma^{k}[i]\right)=\frac{1}{\alpha^{k}} \mu_{\Omega}([i])=\frac{1}{C} \mu_{\mathcal{F}}\left(\varphi\left(S^{n} \sigma^{k}[i]\right)\right) .
$$

As $\varphi$ is continuous and $\mathcal{F}$ is the image of this map, the formula $\mu_{\mathcal{F}}(\varphi B)=C \mu_{\Omega}(B)$, means that $\mu_{\Omega}$ is proportional to the induced measure on $\mathcal{F}$. of $\Omega$ :

We deduce from this that $\varphi$ is almost everywhere one-to-one on each cylinder $[i]$

Proposition 4.4. If $\sigma$ is of Pisot type and unimodular, the representation map $\varphi$ is one-to-one almost everywhere on each cylinder $[i]$ : there exists a subset $\mathcal{N} \subset \Omega$ of measure zero such that

$$
w_{1}, w_{2} \in[i] \backslash \mathcal{N}, \quad \varphi\left(w_{1}\right)=\varphi\left(w_{2}\right) \quad \Longrightarrow \quad w_{1}=w_{2} .
$$


Proof. From Corollary 4.2 the sets

$$
\mathcal{N}_{i}=\bigcup_{\substack{j \neq k \text { or }(p, i, t) \neq(q, i, u) \\ \sigma(j)=p i t, \sigma(k)=q i u}} \varphi\left(S^{|p|} \sigma[j]\right) \cap \varphi\left(S^{|q|} \sigma[k]\right)
$$

have measure zero in $\mathcal{F}$. Thus, by Proposition 4.3, the sets $\mathcal{M}_{i}=\varphi^{-1}\left(\mathcal{N}_{i}\right)$ have measure zero in $\Omega$, and the same is true for

$$
\mathcal{N}=\left\{S^{n}(w), \forall n \in \mathbb{Z}, \exists l \in \mathbb{N}, \sigma^{\mathrm{l}}(w)=w\right\} \bigcup_{j \leq d, k \geq 0, n<\left|\sigma^{k}(j)\right|} S^{n} \sigma^{k} \mathcal{M}_{j} .
$$

Let $w_{1} \neq w_{2} \in[i]$ such that $\varphi\left(w_{1}\right)=\varphi\left(w_{2}\right)$. We have to prove that $w_{1}$ and $w_{2}$ are then in $\mathcal{N}$.

There exists unique $(p, i, t),(q, i, u), j$ and $k$ such that $w_{1} \in S^{|p|} \sigma[j]$ and $w_{2} \in$ $S^{|q|} \sigma[k]$. If $(p, i, t) \neq(q, i, u)$ or $j \neq k, w_{1}$ and $w_{2}$ belong to $\varphi^{-1}\left(\mathcal{N}_{i}\right) \subset \mathcal{N}$. Otherwise, $w_{1}$ and $w_{2}$ satisfy $w_{1}=S^{|p|} \sigma\left(w_{1}^{\prime}\right)$ and $w_{2}=S^{|p|} \sigma\left(w_{2}^{\prime}\right)$, with $w_{1}^{\prime}, w_{2}^{\prime} \in$ $\left[i_{1}\right]$. This implies that $\varphi\left(w_{1}^{\prime}\right)=\varphi\left(w_{2}^{\prime}\right)$. Applying this calculation an infinite number of times, we obtain that $w_{1}$ and $w_{2}$ belong to $\mathcal{N}$ or they have the same prefix-suffix development. But the development map $\Gamma$ is one-to-one everywhere off the orbit of the periodic points of $\sigma$, which is contained in $\mathcal{N}$. Thus, $w_{1}$ and $w_{2}$ belong to $\mathcal{N}$.

For every $x \in \mathcal{F}$, let $n_{x}$ denote the number of points $w$ in $\Omega$ such that $\varphi(w)=x$. An easy consequence of the injectivity on cylinders is that for almost every $x \in \mathcal{F}$, we have $n_{x} \leq d$. Propositions 4.3 and 4.4 mean that the induced measure $\varphi_{\star} \mu_{\Omega}$ has density $C n_{x}$ for the Lebesgue measure $\mu_{\mathcal{F}}$.

In [16], C. Holton and L. Zamboni give other sufficient conditions for injectivity on cylinders.

4.2. Pisot unimodular case with coincidences. Propositions 4.3 and 4.4 also mean that the injectivity in measure of the map $\varphi$ can be expressed in terms of measures of a finite number of sets:

Corollary 4.5. The injectivity in measure of the representation map $\varphi$ is equivalent to the fact that the images of cylinders overlap on a set of measure zero.

For substitutions on two letters, B. Host [18] and C. Holton [14] have proved independently that if a geometric representation map is measure-theoretically oneto-one, then the substitution $\sigma$ satisfies the following coincidence condition.

Definition 4.6. A substitution $\sigma$ satisfies the coincidence condition on prefixes (respectively suffixes) if for every couple of letters $(j, k)$, there exists a constant $n$ such that $\sigma^{n}(j)$ and $\sigma^{n}(k)$ can be decomposed in the following way:

$$
\begin{gathered}
\sigma^{n}(j)=\text { pit and } \sigma^{n}(k)=q i r, \text { with } \mathbf{l}(p)=\mathbf{l}(q) \\
(\text { respectively } \mathbf{l}(t)=\mathbf{l}(u)) .
\end{gathered}
$$

This condition was introduced by F. M. Dekking in [10] for substitutions of constant length and generalized to all substitutions by P. Arnoux and S. Ito in [2]. It is unknown, even in the case $d=2$, if every substitution of Pisot type satisfies the coincidence condition. 
Proposition 4.7. If $\sigma$ is of Pisot type, unimodular and satisfies the coincidence condition, then the representation map $\varphi$ is one-to-one almost everywhere: there exists a subset $\mathcal{N}_{0} \in \Omega$ of measure zero such that

$$
w_{1}, w_{2} \in \Omega \backslash \mathcal{N}_{0}, \quad \varphi\left(w_{1}\right)=\varphi\left(w_{2}\right) \quad \Longrightarrow \quad w_{1}=w_{2} .
$$

Proof. Fix $(j, k)$ in $\mathcal{A} \times \mathcal{A}$. By hypothesis, there exists $i$ such that $\sigma^{n}(j)=$ pit and $\sigma^{n}(k)=q i u$, with $\mathbf{l}(p)=\mathbf{l}(q)$ or $\mathbf{l}(t)=\mathbf{l}(u)$. According to Corollary 4.2 $\varphi\left(S^{|p|} \sigma^{n}[j]\right) \cap \varphi\left(S^{|q|} \sigma^{n}[k]\right)$ has measure zero.

If the coincidence condition is on the prefixes, the two vectors $\delta(p)$ and $\delta(q)$ are equal. Hence, the set $\varphi\left(S^{|p|} \sigma^{n}[j]\right) \cap \varphi\left(S^{|q|} \sigma^{n}[k]\right)$ of measure zero is equal to

$$
\begin{aligned}
\varphi\left(S^{|p|} \sigma^{n}[j]\right) \cap \varphi\left(S^{|q|} \sigma^{n}[k]\right) & =\left(\delta(p)+\mathbf{M}_{\text {diag }}^{n} \varphi[j]\right) \cap\left(\delta(q)+\mathbf{M}_{\text {diag }}^{n} \varphi[k]\right) \\
& =\delta(p)+\mathbf{M}_{\text {diag }}^{n}(\varphi[j] \cap \varphi[k]) .
\end{aligned}
$$

Since the Lebesgue measure is invariant under translation and $\mathbf{M}_{\text {diag }}$ is a linear map of determinant $\pm 1 / \alpha$, we deduce that

$$
\begin{aligned}
0 & =\mu_{\mathcal{F}}\left(\varphi\left(S^{|p|} \sigma^{n}[j]\right) \cap \varphi\left(S^{|q|} \sigma^{n}[k]\right)\right)=\mu_{\mathcal{F}}\left(\delta(p)+\mathbf{M}_{\text {diag }}^{n}(\varphi[j] \cap \varphi[k])\right) \\
& =\left(1 / \alpha^{n}\right) \mu_{\mathcal{F}}(\varphi[j] \cap \varphi[k])=\left(1 / \alpha^{n}\right) \mu_{\Omega}\left(\varphi^{-1}(\varphi[j] \cap \varphi[k])\right) .
\end{aligned}
$$

Let $\mathcal{N}$ be the subset of measure zero defined in Proposition 4.4. Then the following subset $\mathcal{N}_{0}$ of $\Omega$ is of measure zero:

$$
\mathcal{N}_{0}=\mathcal{N} \bigcup_{j \neq k} \varphi^{-1}(\varphi[j] \cap \varphi[k])
$$

Let $w_{1}, w_{2} \in \Omega$ such that $\varphi\left(w_{1}\right)=\varphi\left(w_{2}\right)$. Then, if $\left(w_{1}\right)_{0}=\left(w_{2}\right)_{0}$, by definition of $N, w_{1}$ and $w_{2}$ belong to $N$. Otherwise, $w_{1}$ and $w_{2}$ are in $\varphi^{-1}\left(\varphi\left[\left(w_{1}\right)_{0}\right] \cap \varphi\left[\left(w_{2}\right)_{0}\right]\right)$. In each case, $w_{1}$ and $w_{2}$ belong to $\mathcal{N}_{0}$, that is to say $\varphi$ is one-to-one off a subset of measure zero.

However, if the coincidence condition is on the suffixes, the vectors $\delta(t)$ and $\delta(u)$ are equal. Similar arguments than before lead to the calculation:

$$
\begin{aligned}
0 & =\mu_{\mathcal{F}}\left(\varphi S^{|p|} \sigma^{n}[j] \cap \varphi S^{|q|} \sigma^{n}[k]\right) \\
& =\mu_{\mathcal{F}}\left(\left(\delta(p)+\varphi \sigma^{n}[j]\right) \cap\left(\delta(q)+\varphi \sigma^{n}[k]\right)\right) \\
& =\mu_{\mathcal{F}}\left(\left(\delta\left(\sigma^{n}(j)\right)-\delta(s)+\varphi \sigma^{n}[j]\right) \cap\left(\delta\left(\sigma^{n}(k)\right)-\delta(r)+\varphi \sigma^{n}[k]\right)\right) \\
& =\mu_{\mathcal{F}}\left(-\delta(s)+\left(\delta\left(\sigma^{n}(j)\right)+\varphi \sigma^{n}[j]\right) \cap\left(\delta\left(\sigma^{n}(k)\right)+\varphi \sigma^{n}[k]\right)\right) \\
& =\mu_{\mathcal{F}}\left(\left(\delta\left(\sigma^{n}(j)\right)+\varphi \sigma^{n}[j]\right) \cap\left(\delta\left(\sigma^{n}(k)\right)+\varphi \sigma^{n}[k]\right)\right) \\
& =\mu_{\mathcal{F}}\left(\varphi S^{\left|\sigma^{n}(j)\right|} \sigma^{n}[j] \cap \varphi S^{\left|\sigma^{n}(k)\right|} \sigma^{n}[k]\right)=\mu_{\mathcal{F}}\left(\varphi \sigma^{n} S[j] \cap \varphi \sigma^{n} S[k]\right) \\
& =\left(1 / \alpha^{n}\right) \mu_{\mathcal{F}}(\varphi S[j] \cap \varphi S[k])=\left(1 / \alpha^{n}\right) \mu_{\Omega}\left(S^{-1} \varphi^{-1}(\varphi S[j] \cap \varphi S[k])\right) .
\end{aligned}
$$

In this case we consider the set of measure zero defined by

$$
\mathcal{N}_{0}^{\prime}=\mathcal{N} \bigcup_{j \neq k} S^{-1} \varphi^{-1}(\varphi S[j] \cap \varphi S[k]) .
$$

A similar argument yields that $\varphi$ is one-to-one on $\Omega \backslash \mathcal{N}_{0}^{\prime}$. 

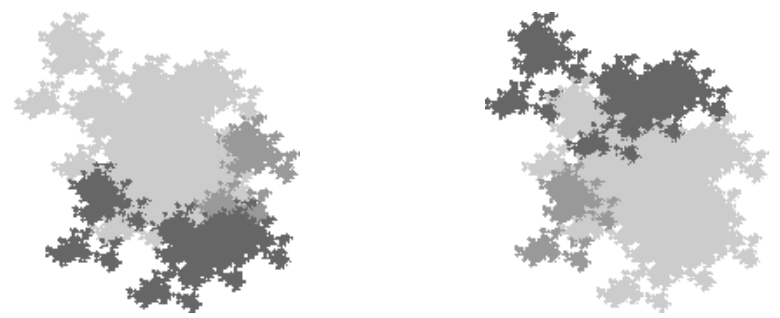

FiguRE 4. Exchange of domains generated by 131,1,1132

4.3. Exchange of domains in the coincidence case. We suppose now that $\sigma$ is of Pisot type, unimodular and satisfies the coincidence condition. The fact that the representation map $\varphi$ is measure-theoretically one-to-one implies that the image of $\Omega$ under $\varphi$ can be decomposed as a disjoint union:

$$
\varphi(\Omega)=\mathcal{F}=\bigcup_{i=1}^{d} \varphi[i] \quad \text { disjoint almost everywhere. }
$$

As a consequence, if we set $\mathcal{F}_{i}=\varphi[i]$, the following exchange of domains $\mathcal{T}$ is defined almost everywhere on $\mathcal{F}$ (see an example in Figure 4):

$$
\begin{aligned}
\mathcal{T}: \mathcal{F} & \rightarrow \mathcal{F} \\
x \in \mathcal{F}_{i} & \mapsto x+\delta(i) .
\end{aligned}
$$

Proposition 2.3 now means that the following diagrams commute:
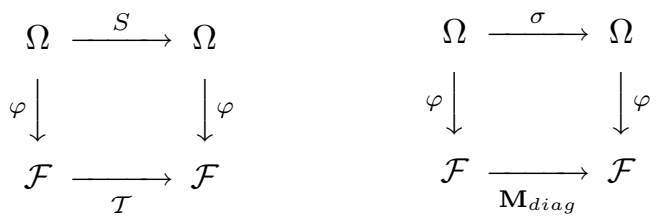

But the set $\mathcal{F}$ has a self-similar structure in the following sense: if for each prefix $p$ we call $h_{p}$ the contracting map defined by

$$
\begin{aligned}
h_{p}: \quad \mathbb{R}^{r-1} \times \mathbb{C}^{s} & \rightarrow \mathbb{R}^{r-1} \times \mathbb{C}^{s} \\
\mathbf{x} & \mapsto \mathbf{M}_{\text {diag }} \mathbf{x}+\delta(p),
\end{aligned}
$$

then the partition of each cylinder on the form $[i]=\bigcup_{\sigma(j)=p i t} S^{|p|} \sigma[j]$ and the injectivity of $\varphi$ imply that the images $\mathcal{F}_{i}$ of cylinders decompose as the following partition, which is disjoint almost everywhere:

$$
\mathcal{F}_{i}=\bigcup_{\sigma(j)=p i t} h_{p}\left(\mathcal{F}_{j}\right) \quad \text { disjoint almost everywhere. }
$$

Thus, the compact set $\mathcal{F}$ is tiled by the self-similar tiles $\mathcal{F}_{i}$ as defined in [21].

This proves that the representation map $\varphi$ is a measure-theoretic isomorphism between $(\Omega, S)$ and the transformation $\mathcal{T}$ on the self-similar set $\mathcal{F}$. As the transformation $p$ is a linear isomorphism from $\mathbb{R}^{r-1} \times \mathbb{C}^{s}$ onto $\mathbb{R}^{d-1}$, we get the following theorem.

Theorem 4.8. If $\sigma$ is a substitution on d letters which is of Pisot type, unimodular and satisfies the coincidence condition, $(\Omega, S)$ is measure-theoretically conjugate to an exchange of domains on a self-similar compact subset of $\mathbb{R}^{d-1}$. 


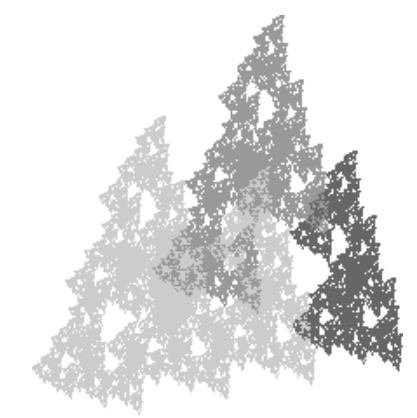

FiguRE 5 . The fractal generated by $12,31,1$

More precisely, the representation map $p_{\mathcal{H}} \circ \varphi$ is continuous from $\Omega$ onto its image $p_{\mathcal{H}}(\mathcal{F})$, one-to-one off a subset of $\Omega$ of measure zero, and realizes a conjugation between the shift map on $\Omega$ and an exchange of domains defined almost everywhere on $p_{\mathcal{H}}(\mathcal{F})$.

Example 4.9 (see Figure 5). Let $\sigma$ be the substitution $1 \mapsto 12,2 \mapsto 31,3 \mapsto 1$ and $\beta$ be a contracting eigenvalue of $\mathbf{M}_{\sigma}$. The matrix $\mathbf{M}_{\text {diag }}$ is simply $(\beta)$ and the vector $\delta=(\delta(1), \delta(2), \delta(3))$ can be chosen as $\left(1, \beta-1, \beta^{2}-\beta-1\right)$. Then $\mathcal{F}=\mathcal{F}_{1} \cup \mathcal{F}_{2} \cup \mathcal{F}_{3}$ has the following self-similar structure:

$$
\begin{aligned}
& \mathcal{F}_{1}=\beta \mathcal{F}_{1} \cup\left(\beta \mathcal{F}_{2}+\beta^{2}-\beta-1\right) \cup \beta \mathcal{F}_{3}, \\
& \mathcal{F}_{2}=\beta \mathcal{F}_{1}+1, \\
& \mathcal{F}_{3}=\beta \mathcal{F}_{2} .
\end{aligned}
$$

One can remark that formula (4.1) is verified by all substitution of Pisot type if we replace the disjoint union by a simple union. This gives a new proof of a recent result due to V.S. Sirvent and Y. Wang in 31.

Proposition 4.10 ([31]). If $\sigma$ is of Pisot type, the sets $\mathcal{F}_{i}$ have nonempty interior.

Proof. By Corollary 3.9, $\mathcal{F}$ has nonempty interior, so that there exists an integer $i_{0}$ such that $\mathcal{F}_{i_{0}}$ has nonempty interior. The self-similar structure of $\mathcal{F}$ (Formula (4.11)), and the fact that the prefix-suffix automaton is strongly connected imply that each $\mathcal{F}_{i}$ contains a copy of $\mathcal{F}_{i_{0}}$ : there exists a finite sequence of prefix $p_{1}, \ldots$, $p_{k}$ such that $h_{p_{1}} \ldots h_{p_{k}}\left(\mathcal{F}_{i_{0}}\right) \subset \mathcal{F}_{i}$, so that $\mathcal{F}_{i}$ have nonempty interior.

In [31], the authors also prove that each $\mathcal{F}_{i}$ is the closure of its interior.

\section{Suffixes CONSTRuCtion}

The representation map $\varphi$ that we defined takes only into account the prefixes $p_{i}$ of the prefix-suffix development of points of $\Omega$. It is also possible, as in [28] to define a representation map with the suffixes of this development:

$$
\forall w \in \Omega \quad \varphi^{\prime}(w)=\sum_{i \geq 0} \mathbf{M}_{\text {diag }}^{j} \delta\left(s_{i}\right) \in \mathbb{R}^{r-1} \times \mathbb{C}^{s} \quad \text { with } \Gamma(w)=\left(p_{i}, a_{i}, s_{i}\right)_{i \geq 0} .
$$

Formula (2.4) implies that the maps $\varphi$ and $\varphi^{\prime}$ are linked by the relation

$$
\forall w \in \Omega \quad \varphi^{\prime}(w)=-\varphi(w)-\delta\left(w_{0}\right)=-\varphi(S w) .
$$


So that the prefix and suffix constructions are completely equivalent. This is coherent if one considers the fact that every substitutive system has zero entropy: thus, knowledge of the past of a point of $\Omega$, that is its negative part described by $\varphi$, is "equivalent" to knowledge of the future, that is its positive part described by $\varphi^{\prime}$.

\section{REFERENCES}

1. P. Arnoux, Recoding sturmian sequences on a subshift of finite type. Chaos from order, a worked out example, 1998 FIESTA Summer School, December 1998, Chili.

2. P. Arnoux and S. Ito, Pisot substitutions and Rauzy fractals, Preprint 98-18, Institut de Mathématiques de Luminy, 1998.

3. P. Arnoux and G. Rauzy, Représentation géométrique de suites de complexité $2 n+1$, Bull. Soc. Math. France 119 (1991), no. 2, 199-215. MR 92k:58072

4. A. Berman and R. J. Plemmons, Nonnegative matrices in the mathematical sciences, SIAM, Philadelphia, PA, 1994; revised reprint of 1979 original. MR 95e:15013

5. E. Bombieri and J. E. Taylor, Which distributions of matter diffract? An initial investigation, J. Physique 47 (1986), no. 7 Suppl. Colloq. C3, C3-19-C3-28, International workshop on aperiodic crystals (Les Houches, 1986). MR 88a:52015

6. M. Boshernitzan and I. Kornfeld, Interval translation mappings, Ergodic Theory Dynam. Systems 15 (1995), no. 5, 821-832. MR 96m:58068

7. V. Canterini, Géométrie des substitutions pisot unitaires, Ph.D. thesis, Université de la Méditerranée, 2000.

8. V. Canterini and A. Siegel, Automate des préfixes-suffixes associé à une substitution primitive, To appear in J. Théor. Nombres Bordeaux.

9. J. Cassaigne, S. Ferenczi, and L. Q. Zamboni, Imbalances in Arnoux-Rauzy sequences, Ann. Inst. Fourier 50 (2000), no. 4, 1265-1276. CMP 2001:05

10. F. M. Dekking, The spectrum of dynamical systems arising from substitutions of constant length, Z. Wahrscheinlichkeitstheorie und Verw. Gebiete 41 (1978), no. 3, 221-239. MR 57:1455

11. Recurrent sets, Adv. in Math. 44 (1982), no. 1, 78-104. MR 84e:52023

12. S. Ferenczi, Les transformations de Chacon: combinatoire, structure géométrique, lien avec les systèmes de complexité $2 n+1$, Bull. Soc. Math. France 123 (1995), no. 2, 271-292. MR 96m:28018

13. M. Hollander, Linear numeration systems, finite $\beta$-expansions, and discrete spectrum of substitution dynamical systems, Ph.D. thesis, University of Washington, 1996.

14. C. Holton, Private communication, 1999.

15. C. Holton and L. Q. Zamboni, Geometric realizations of substitutions, Bull. Soc. Math. France 126 (1998), no. 2, 149-179. MR 2000c:37008

16. _ Directed graphs and substitutions, Preprint, 1999.

17. B. Host, Valeurs propres des systèmes dynamiques définis par des substitutions de longueur variable, Ergodic Theory Dynamical Systems 6 (1986), no. 4, 529-540. MR 88i:26007

18. $\ldots$ Représentation géométrique des substitutions sur 2 lettres, unpublished manuscript, 1992.

19. S. Ito and M. Kimura, On Rauzy fractal, Japan J. Indust. Appl. Math. 8 (1991), no. 3, 461-486. MR 93d:11084

20. A. Katok and B. Hasselblatt, Introduction to the modern theory of dynamical systems, Cambridge University Press, Cambridge, 1995. MR 96c:58055

21. R. Kenyon, Self-similar tilings, Ph.D. thesis, Princeton University, 1990.

22. R. Kenyon and A. Vershik, Arithmetic construction of sofic partitions of hyperbolic toral automorphisms, Ergodic Theory Dynam. Systems 18 (1998), no. 2, 357-372. MR 99g:58092

23. A. N. Livshits, Some examples of adic transformations and automorphisms of substitutions, Selecta Math. Soviet. 11 (1992), no. 1, 83-104, Selected translations. MR 93d:28024

24. A. Messaoudi, Autour du fractal de rauzy, Ph.D. thesis, Université de la Méditerranée, 1996.

25. B. Mossé, Reconnaissabilité des substitutions et complexité des suites automatiques, Bull. Soc. Math. France 124 (1996), no. 2, 329-346. MR 97k:11030

26. M. Queffélec, Substitution dynamical systems - spectral analysis, Lecture Notes in Mathematics, 1294. Springer-Verlag, Berlin, 1987. MR 89g:54094 
27. G. Rauzy, Nombres algébriques et substitutions, Bull. Soc. Math. France 110 (1982), no. 2, 147-178. MR 84h:10074

28. _ Rotations sur les groupes, nombres algébriques, et substitutions, Séminaire de Théorie des Nombres, 1987-1988 (Talence, 1987-1988), Univ. Bordeaux I, Talence, 1988, Exp. No. 21. MR 90g:11017

29. A. Siegel, Facteurs p-adiques des substitutions primitives non unitaires, Preprint, 2000.

30. - Représentations géométrique, combinatoire et arithmétique des systèmes substitutifs de type Pisot, Ph.D. thesis, Université de la Méditérannée, 2000.

31. V. Sirvent and Y. Wang, Geometry of the Rauzy Fractals, Preprint, 1999.

32. B. Solomyak, On the spectral theory of adic transformations, Representation theory and dynamical systems, Amer. Math. Soc., Providence, RI, 1992, pp. 217-230. MR 93h:28028

33. M. Solomyak, On simultaneous action of Markov shift and adic transformation, Representation theory and dynamical systems, Amer. Math. Soc., Providence, RI, 1992, pp. 231-239. MR 93h:28029

Institut de Mathématiques de Luminy, UPR 9016, Case 907, 163 avenue de Luminy, 13288 Marseille Cedex 9, France

Current address: CESAME, Université Catholique de Louvain, Bâtiment Euler, Avenue G. Lemairè, 4, 1348 Louvain-la-Neuve, Belgium

E-mail address: canterini@anma.ucl.ac.be

Institut de Mathématiques de Luminy, UPR 9016, Case 907, 163 avenue de Luminy, 13288 Marseille Cedex 9, France

E-mail address: siegel@iml.univ-mrs.fr 